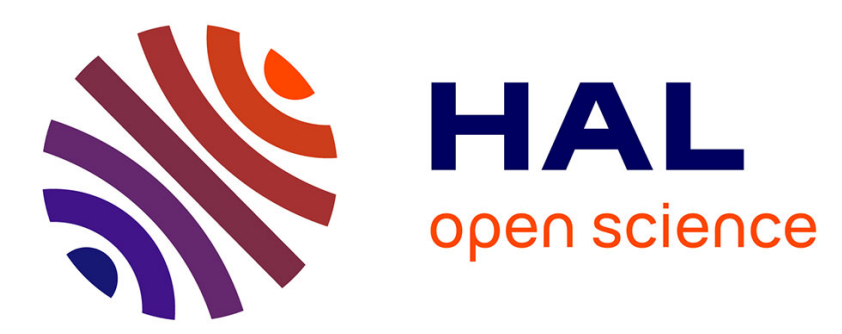

\title{
Prox-regularity approach to generalized equations and image projection
}

\author{
Samir Adly, Florent Nacry, Lionel Thibault
}

\section{To cite this version:}

Samir Adly, Florent Nacry, Lionel Thibault. Prox-regularity approach to generalized equations and image projection. ESAIM: Control, Optimisation and Calculus of Variations, 2018, 24 (2), pp.677-708. $10.1051 / \mathrm{cocv} / 2017052$. hal-02072430

\section{HAL Id: hal-02072430 \\ https://hal.science/hal-02072430}

Submitted on 20 Oct 2020

HAL is a multi-disciplinary open access archive for the deposit and dissemination of scientific research documents, whether they are published or not. The documents may come from teaching and research institutions in France or abroad, or from public or private research centers.
L'archive ouverte pluridisciplinaire HAL, est destinée au dépôt et à la diffusion de documents scientifiques de niveau recherche, publiés ou non, émanant des établissements d'enseignement et de recherche français ou étrangers, des laboratoires publics ou privés. 


\title{
PROX-REGULARITY APPROACH TO GENERALIZED EQUATIONS AND IMAGE PROJECTION
}

\author{
SAmir Adly ${ }^{1, a}$, Florent NaCRY $^{2}$ And Lionel Thibault ${ }^{3}$
}

\begin{abstract}
In this paper, we first investigate the prox-regularity behaviour of solution mappings to generalized equations. This study is realized through a nonconvex uniform Robinson-Ursescu type theorem. Then, we derive new significant results for the preservation of prox-regularity under various and usual set operations. The role and applications of prox-regularity of solution sets of generalized equations are illustrated with dynamical systems with constraints.
\end{abstract}

\section{INTRODUCTION}

\subsection{Context of the paper}

In his famous paper "Curvature measures" [15] Federer defined a closed subset $S$ in $\mathbb{R}^{n}$ to be positively reached if $\operatorname{reach}(S)>0$, where $\operatorname{reach}(S)$ is the supremum of all reals $r>0$ such that any $x \in \mathbb{R}^{n}$ with $\operatorname{dist}(x, S)<r$ has a unique nearest point in $S$. For a positively reached set in $\mathbb{R}^{n}$ and for $S_{r}:=\left\{x \in \mathbb{R}^{n}: \operatorname{dist}(x, S) \leq r\right\}$, it is shown in [15] that the Lebesgue measure of $S_{r}$ is a polynomial of degree $n$ for $0 \leq r<\operatorname{reach}(S)$. This was a remarkable extension to positively reached sets of Steiner polynomial formula previously known only for convex sets or $C^{2}$ submanifolds. The approach used in [15] required a "Principal Kinematic Formula", and this was one of the motivations which led Federer to establish what is known as "Coarea Formula". This has been proved by Federer in Theorem 3.1 in page 9 in [15]. The term "Coarea Formula" has been coined by Federer in the page 241 of his book [16]. In a very large recent survey [40], one can find many summarized results on various aspects of positively reached sets related either to differential geometry or to geometric measure theory.

In the context of (infinite dimensional) Hilbert space $\mathcal{H}$ the concept of $r$-prox-regularity for a closed subset $S$ in $\mathcal{H}$ has been defined via the variational notion of proximal normal and it has been proved that $S$ is $r$-prox-regular $(r \in] 0,+\infty])$ if and only if the metric projection is single-valued and continuous on the set $\{x \in \mathcal{H}: \operatorname{dist}(x, S)<r\}$. So, a closed convex subset in $\mathcal{H}$ is evidently $r$-prox-regular with $r=+\infty$; further, in

Keywords and phrases. Variational analysis, prox-regular set, metric regularity, generalized equation, Robinson-Ursescu Theorem, variational inclusion, nonsmooth dynamics.

1 Laboratoire XLIM, Université de Limoges, 123, Avenue Albert Thomas, 87060 Limoges, Cedex, France.

a Corresponding author: samir.adly@unilim.fr;

florent.nacry@unilim.fr

2 Département de Mathématiques, Université Montpellier, 34095 Montpellier Cedex 5, France.

lionel.thibault@univ-montp2.fr

3 Centro de Modelamiento Matematico, Universidad de Chile. 
finite dimensional Euclidean space it is clear that a set is prox-regular if and only if it is positively reached. The prox-regularity also appears in the literature under different names (weakly convex sets, proximally smooth sets) and it is well established that it plays an important role in many aspects in both pure and applied mathematics (see, e.g., $[1,9,10,12,15,24,36,40]$ and the references therein).

Under the prox-regularity of the moving subset $C(t) \subset \mathcal{H}, t \in[0, T]$, the fundamental dynamical differential evolution system (known as "Perturbed Moreau's Sweeping Process")

$$
N(C(t) ; u(t))+f(t, u(t)) \ni 0 \quad \text { with } \quad u(0)=u_{0} \in C(0),
$$

is known to admit, under natural Lipschitz assumptions on $C(\cdot)$ and $f(t, \cdot)$ for example, one and only one solution (see, e.g., $[1,12]$ and references therein). This is an important extension to the prox-regular context of a previous result with convex sets $C(t)$. The above dynamical system with prox-regular sets has been recently used by Maury and Venel [24] and Maury [25] for the modeling of crowd motion (in both discrete and continuous dynamics). More precisely, the problem considers $N$ persons in a room, identified to planar balls with radius $r>0$ and center $x_{i}, i=1,2, \ldots, N$. Overlapping between persons being not allowed, the vector position $x=\left(x_{1}, x_{2}, \ldots, x_{N}\right) \in \mathbb{R}^{2 N}$ is required to belong to the feasible set of configurations $S=\left\{x \in \mathbb{R}^{2 N}\right.$ : $\left.\left\|x_{i}-x_{j}\right\|-2 r \geq 0, \forall i \neq j\right\}$, which is showed to be prox-regular while it is nonconvex (see [24] for more details). The authors involved in [24] the dynamical system (1.1) with the above prox-regular set $S$ as $C(t)$.

It is also worth pointing out that a strong link with Von Neumann alternating projection algorithm and prox-regularity exists since several authors have studied such algorithms in the framework of prox-regular sets see for example, [22] and references therein. Other domains where prox-regularity is required will be presented in the next section. The class of prox-regular sets is much larger than the class of nonempty closed convex sets and shares with it many nice properties, as the differentiability of squared distance function in some enlargement of the set. However, unlike the convexity property, prox-regularity is not preserved by set operations (as intersection) and this leads to find conditions ensuring such a property $[2,12,42,43]$.

The present paper is devoted to the study of prox-regularity of the set of solutions of various generalized equations in the form $F(x) \ni 0$ in Hilbert space $\mathcal{H}$, that is, the set $\{x \in \mathcal{H}: F(x) \ni 0\}$, where $F$ is a multimapping from $\mathcal{H}$ into another Hilbert space. This will be seen to contain the intersection as a particular case.

\subsection{Contributions of the paper}

In fact, we are interested in the prox-regularity of the solution set of generalized equations in the form

$$
F_{1}(x)+F_{2}(x) \ni 0
$$

where $F_{1}, F_{2}: \mathcal{H} \rightrightarrows \mathcal{H}^{\prime}$ are two multimappings between Hilbert spaces. Our aim is to develop sufficient verifiable conditions guaranteeing the prox-regularity of the solution set $\left(F_{1}+F_{2}\right)^{-1}(0)$; our main result in this direction is Theorem 6.5. The key of our approach is a suitable extension of the famous Robinson-Ursescu Theorem [35,41], which asserts for two Banach spaces $X$ and $Y$, a multimapping $M: X \rightrightarrows Y$ with a closed convex graph is metrically regular at $\bar{x}$ for $\bar{y} \in M(X)$ if and only if $\bar{y} \in$ int $M(X)$. Over the years, many researchers have weakened the convexity assumption in Robinson-Ursescu theorem (see, e.g., [17, 18, 44, 47]). Our result is concerned with the class of normally $\omega(\cdot)$-regular sets, a new class which encompasses prox-regular sets in the Hilbert setting and $(\sigma, \delta)$-subsmooth sets [44] in a general Banach space. From the prox-regularity of $\left(F_{1}+F_{2}\right)^{-1}(0)$, we derive new significant results for the study of generalized equations, inverse and direct images, intersections and constrained sets. Other general results are obtained for the case of the projection along a linear space of prox-regular sets.

\subsection{Organization of the paper}

The paper is organized as follows. We begin in Section 2 with an application of Theorem 6.5 to a selection principle for a nonsmooth dynamical system whose constraint is the set of solutions of a generalized equation. 
Section 3 is devoted on one hand to the notation and the necessary preliminaries needed in the rest of paper. In Section 4, we recall basic facts concerning prox-regularity and we introduce the new concept of normal $\omega(\cdot)$ regularity. In Section 5, we first provide properties and counterexamples for multimappings with prox-regular graphs. Then, we give, as our first main result, a Robinson-Ursescu type theorem for multimappings with normally $\omega(\cdot)$-regular graphs. In Section 6 , we focus on the prox-regularity of the solution set of generalized equations and we prove Theorem 6.5 on prox-regularity of generalized equations in the form $F_{1}(x)+F_{2}(x) \ni 0$, the second main result of the paper. In Section 7, we develop new results for the preservation of prox-regularity under set operations and we provide an example (see Exp. 7.12) of a prox-regular set whose image under a coordinate projector is not prox-regular.

\section{Application to nonsmooth Dynamics}

We start with an application of Theorem 6.5 to nonsmooth dynamical systems. Consider a generalized equation in the form

$$
F(x) \ni 0 \quad x \in \mathcal{H},
$$

where $F: \mathcal{H} \rightrightarrows \mathcal{H}^{\prime}$ is a multimapping between two Hilbert spaces, and denote by $S:=\{x \in \mathcal{H}: F(x) \ni$ $0\}=F^{-1}(0)$ its set of solutions. Generally, (2.1) admits infinitely many solutions. In such a case, a challenging fundamental question arises: How to select or construct a solution which is the unique one for which certain basic properties are required? When $S=F^{-1}(0)$ is convex and closed, one principle for selecting such a solution is to require that it minimizes the squared norm function $\frac{1}{2}\|\cdot\|^{2}$ among all the solutions: of course, such a solution exists and is unique according to the convexity and closedness property of $S$, and it is nothing else the solution of minimal norm. Under the convexity (and closedness) of $S$, several other principles are also known: The dynamical selection principle is one of the most efficient. Given a smooth convex function $g: \mathcal{H} \rightarrow \mathbb{R}$ (whose derivative is locally Lipschitz continuous) and a point $x_{0} \in S$, the principle associates the dynamical system

$$
\left\{\begin{array}{l}
-\frac{\mathrm{d} x}{\mathrm{~d} t}(t) \in \nabla g(x(t))+N\left(F^{-1}(0) ; x(t)\right) \quad \text { a.e. } t \in[0,+\infty[ \\
x(0)=x_{0}
\end{array}\right.
$$

which is known to possess a unique (locally absolutely continuous) solution $x(\cdot)$. Further, it is also known that the solution $x(\cdot)$ of $(2.2)$ converges at $+\infty$, say $\lim _{t \rightarrow+\infty} x(t)=: x_{\infty}$, which furnishes a selected solution of $(2.1)$.

Going beyond the convexity of $F^{-1}(0)$ and of the smooth function $g$, we will show that the prox-regularity of the set $S=F^{-1}(0)$ will allow us to obtain the existence and uniqueness of a trajectory of (2.2), for a given initial point $x_{0}$. We will also show (under a Palais-Smale condition) that this trajectory converges to a point $x_{\infty} \in S=F^{-1}(0)$ that satisfies, through the normal cone $N\left(F^{-1}(0) ; \cdot\right)$, the following principle selection

$$
0 \in \nabla g\left(x_{\infty}\right)+N\left(F^{-1}(0) ; x_{\infty}\right),
$$

which can be regarded as the first order optimality condition of the following constrained optimization problem

$$
\left\{\begin{array}{l}
\text { Minimize } g(x) \\
\text { subject to the constraint } \quad 0 \in F(x) .
\end{array}\right.
$$

For the particular choice of $g=\frac{1}{2}\|\cdot\|^{2}$ and when $F^{-1}(0)$ is a nonempty $r$-prox-regular set with $d\left(0, F^{-1}(0)\right)<r$, the point $x_{\infty}$ is equal to $P_{F^{-1}(0)}(0)$ (the unique metric projection of 0 onto $F^{-1}(0)$ ), that is, it corresponds to the element of minimal norm of $F^{-1}(0)$ (known also in the literature as the lazy selector); this is the aforementioned minimal norm solution. 
In order to state the nonconvex result concerning (2.2) and (2.3), it will be convenient for us to say that the function $g: \mathcal{H} \rightarrow \mathbb{R}$ satisfies the generalized Palais-Smale condition relative to a uniformly prox-regular subset $C$ of $\mathcal{H}$ whenever each sequence $\left(x_{n}\right)_{n \in \mathbb{N}}$ of $C$ such that

$$
\sup _{n \in \mathbb{N}} g\left(x_{n}\right)<+\infty \quad \text { and } \quad d\left(0, \nabla g\left(x_{n}\right)+N\left(C ; x_{n}\right)\right) \rightarrow 0 \text { as } n \rightarrow+\infty
$$

has a convergent subsequence in $\mathcal{H}$.

Proposition 2.1. Let $\mathcal{H}, \mathcal{H}^{\prime}$ be Hilbert spaces, $F: \mathcal{H} \rightrightarrows \mathcal{H}^{\prime}$ be a multimapping. Let $g: \mathcal{H} \rightarrow \mathbb{R}$ be a $C^{1,1}$ function bounded from below. Assume that:

(i) $F^{-1}(0)$ is uniformly prox-regular, which is the case under the assumptions of Theorem 6.5 with $F_{1} \equiv 0$ and $F_{2}=F$;

(ii) the mapping $g$ satisfies the generalized Palais-Smale condition (2.4) relative to $F^{-1}(0)$.

Then, for each $x_{0} \in F^{-1}(0)$, there exists a unique locally absolutely continuous solution $x:\left[0,+\infty\left[\rightarrow F^{-1}(0)\right.\right.$ of the dynamical system

$$
\left\{\begin{array}{l}
-\frac{\mathrm{d} x}{\mathrm{~d} t}(t) \in \nabla g(x(t))+N\left(F^{-1}(0) ; x(t)\right) \quad \text { a.e. } \quad t \in[0,+\infty[ \\
x(0)=x_{0} .
\end{array}\right.
$$

Further, the set $\bigcap_{T \geq 0} \operatorname{cl}\left(x\left([T,+\infty[))\right.\right.$ is nonempty and for any $x_{\infty} \in \bigcap_{T \geq 0} \operatorname{cl}(x([T,+\infty[))$, one has

$$
\lim _{t \rightarrow+\infty} g(x(t))=g\left(x_{\infty}\right)=\inf _{t \geq 0} g(x(t))
$$

as well as the first order optimality condition

$$
0 \in \nabla g\left(x_{\infty}\right)+N\left(F^{-1}(0) ; x_{\infty}\right)
$$

A basic selected solution for (2.1) is then obtained.

Proof. Set $S:=F^{-1}(0)$ (as above) and $h:=g+\psi_{S}$, where $\psi_{S}$ denotes the indicator function of $S$ (in the sense of variational analysis, i.e., $\psi_{S}(x)=0$ if $x \in S$ and $\psi_{S}(x)=+\infty$ if $x \in \mathcal{H} \backslash S$ ). Obviously, note that $h$ is bounded from below and proper. Combining the uniform prox-regularity of $S$ with the fact that $g$ is a $C^{1,1}$ function, it can be easily checked that $h$ is primal lower nice (see [30] for the definition) at each point of $S$. Fix any $x_{0} \in S$. Applying ([23], Thm. 3.2), there is one and only one locally absolutely continuous mapping $x:\left[0,+\infty\left[\rightarrow F^{-1}(0)\right.\right.$ satisfying

$$
\left\{\begin{array}{l}
-\frac{\mathrm{d} x}{\mathrm{~d} t}(t) \in \partial_{C} h(x(t)) \quad \text { a.e. } t \in[0,+\infty[ \\
x(0)=x_{0}
\end{array}\right.
$$

By the sum rule of the Clarke subdifferential $\partial_{C} h$ (keeping in mind that $g$ is $C^{1,1}$ ), the latter inclusion means that, for almost every $t \in[0,+\infty[$,

$$
-\frac{\mathrm{d} x}{\mathrm{~d} t}(t) \in \nabla g(x(t))+N(S ; x(t)) .
$$

Given $x_{\infty} \in \bigcap_{T \geq 0} \operatorname{cl}(x([T,+\infty[))$, it remains to invoke ([23], Prop. 4.5) to obtain that it satisfies both (2.6) and (2.7). 
It is worth pointing out that an adaptation of the catching-up algorithm introduced by Moreau gives a way to construct explicitly the unique solution $x:[0,+\infty[\rightarrow \mathcal{H}$ of $(2.5)$, that is, proceed to a time discretization $\left(t_{i}\right)_{i \in I}$ of $[0,+\infty[$ and set

$$
\left\{\begin{array}{l}
x_{0}^{n}:=x_{0} \\
x_{i+1}^{n}:=\operatorname{proj}_{S}\left[x_{i}^{n}-\left(t_{i+1}^{n}-t_{i}^{n}\right) \nabla g\left(x_{i}^{n}\right)\right] \quad \text { for all } \quad i \in I .
\end{array}\right.
$$

Thanks to the prox-regularity of $S=F^{-1}(0)$, the above scheme is well defined for suitable time discretizations $\left(t_{i}\right)_{i \in I}$.

Lur'e dynamical system in the next example is a basic situation for application of Proposition 2.1.

Example 2.2. Given two integers $m, n \geq 1$, two matrices $A \in \mathbb{R}^{n \times n}, B \in \mathbb{R}^{m \times n}$ with $A$ symmetric and $B$ surjective, a vector $x_{0} \in \mathbb{R}^{n}$ and a multimapping $F: \mathbb{R}^{m} \rightrightarrows \mathbb{R}^{n}$, one can consider with $S:=F^{-1}(0) \subset \mathbb{R}^{m}$, the problem of finding a locally absolutely continuous mapping $x:\left[0,+\infty\left[\rightarrow \mathbb{R}^{n}\right.\right.$ satisfying the following Lur'e dynamical system

$$
(\mathcal{L})\left\{\begin{array}{llc}
\frac{\mathrm{d} x}{\mathrm{~d} t}(t)=A x(t)-B^{T} \lambda(t) & \text { a.e. } & t \in[0,+\infty[ \\
y(t)=B x(t) & \text { for all } & t \geq 0 \\
\lambda(t) \in N(S ; y(t)) & \text { for all } & t \geq 0 \\
x(0)=x_{0} . & &
\end{array}\right.
$$

Lur'e dynamical systems are of great interest for the modeling of many problems in nonlinear control theory. It consists of a linear differential system combined with a static multivalued nonlinearity $\lambda \in N\left(F^{-1}(0) ; y\right)$. Assume that:

(i) $F^{-1}(0)$ is uniformly prox-regular;

(ii) The mapping $g: \mathbb{R}^{n} \rightarrow \mathbb{R}^{n}$ defined by

$$
g(x):=\frac{1}{2}\langle-A x, x\rangle \quad \text { for all } x \in \mathbb{R}^{n}
$$

is bounded from below and satisfied the Palais-Smale condition relative to $B^{-1}(S)$.

Let $x(\cdot)$ be a solution of $(\mathcal{L})$. It is readily seen that for almost every $t \in[0,+\infty[$,

$$
\frac{\mathrm{d} x}{\mathrm{~d} t}(t) \in A x(t)-B^{T} N(S ; B x(t)) .
$$

Since $B$ has rank $m$, we can apply ([36], Exercise 10.7), to get that for all $x \in \mathbb{R}^{m}$,

$$
\partial_{C}\left(\psi_{B^{-1}(S)}\right)(x)=B^{T} \partial_{C} \psi_{S}(B x)=B^{T} N(S ; B x),
$$

where (as above) $\psi_{S}$ denotes the indicator function of the set $S$.

Consequently, (2.8) is equivalent to

$$
\frac{\mathrm{d} x}{\mathrm{~d} t}(t) \in A x(t)-N\left(B^{-1}(S) ; x(t)\right) \quad \text { a.e. } t \in[0,+\infty[.
$$

We observe that (2.9) can be rewritten as (2.5), that is,

$$
-\frac{\mathrm{d} x}{\mathrm{~d} t}(t) \in \nabla g(x(t))+N\left(B^{-1}(S) ; x(t)\right) \quad \text { a.e. } t \in[0,+\infty[\text {. }
$$


From ([2], Thm. 6.4), we know (keeping in mind that $B$ is surjective) that $B^{-1}(S)$ is uniformly prox-regular. By virtue of Proposition 2.1, there is $\bar{x} \in \mathbb{R}^{n}$ such that $x(t) \rightarrow \bar{x}$ as $t \rightarrow+\infty$ and satisfying the first order optimality condition

$$
0 \in \nabla g(\bar{x})+N\left(B^{-1}(S) ; \bar{x}\right)
$$

for the constrained optimization problem

$$
\left\{\begin{array}{l}
\text { Minimize } \frac{1}{2}\langle A x, x\rangle \\
\text { subject to the constraint } B x \in S .
\end{array}\right.
$$

\section{NOTATION AND PRELIMINARIES}

In the whole paper, all vector spaces are real vector spaces, $\mathbb{R}_{+}:=[0,+\infty[$ is the set of nonnegative reals, $\mathbb{N}$ is the set of positive integers (i.e., $n=1, \ldots)$. We use the classical convention $\inf \emptyset=+\infty$. For a nonempty set $E$ and $m \in \mathbb{N}$, we denote by $\Delta_{E^{m}}$ the diagonal of $E^{m}$, i.e.,

$$
\Delta_{E^{m}}:=\{(x, \ldots, x): x \in E\} \subset E^{m} .
$$

Throughout the rest of the paper, $(X,\|\cdot\|)$ is a Banach space, $X^{*}$ its topological dual and $\langle\cdot, \cdot\rangle$ the associated pairing. For $x \in X$ and $r \in] 0,+\infty[$ one denotes by $B(x, r)$ (resp., $B[x, r]$ ) the open (resp., closed) ball of $X$ centered at $x \in X$ with radius $r \in] 0,+\infty\left[\right.$. It will be convenient to denote by $\mathbb{U}_{X}$ and $\mathbb{B}_{X}$ the open and closed unit balls of $X$ respectively, that is, $\mathbb{U}_{X}:=B(0,1)$ and $\mathbb{B}_{X}:=B[0,1]$.

Let $S$ be a nonempty subset of $X$. This set is said to be closed near $x \in S$ whenever there is a neighborhood $V$ of $x$ such that $S \cap V$ is closed in $V$ with respect to the induced topology on $V$. The distance function from $S$ is defined by

$$
d_{S}(x):=: d(x, S):=\inf _{y \in S}\|x-y\| \quad \text { for all } x \in X .
$$

For another subset $S^{\prime}$ of $X$, the gap between $S$ and $S^{\prime}$ is the extended real

$$
\operatorname{gap}\left(S, S^{\prime}\right):=\inf _{(x, y) \in S \times S^{\prime}}\|x-y\| .
$$

For any $x \in X$, one defines the (possibly empty) set of all nearest points of $x$ in $S$ by

$$
\operatorname{Proj}_{S}(x):=\{y \in S: d(x, S)=\|x-y\|\} .
$$

When $\operatorname{Proj}_{S}(x)$ contains one and only one point $\bar{y}$, we will denote by $P_{S}(x) \operatorname{or}_{\operatorname{proj}_{S}}(x)$ the unique element, that is, $P_{S}(x):=\bar{y}$.

For the subset $S$ of $X, \sigma(S, \cdot)$ stands for its support function, i.e.,

$$
\sigma\left(S ; x^{\star}\right):=\sup _{x \in S}\left\langle x^{\star}, x\right\rangle \quad \text { for all } x^{\star} \in X^{\star} .
$$

Before defining the concepts of prox-regularity and $\omega(\cdot)$-regularity for sets, we need diverse notions of variational analysis.

The Clarke tangent cone of the subset $S$ of $X$ at $x \in S$, denoted by $T^{C}(S ; x)$, is the set of $h \in X$ such that, for every sequence $\left(x_{n}\right)_{n \in \mathbb{N}}$ of $S$ with $x_{n} \rightarrow x$ and for every sequence $\left(t_{n}\right)_{n \in \mathbb{N}}$ of positive reals with $t_{n} \rightarrow 0$, there exists a sequence $\left(h_{n}\right)_{n \in \mathbb{N}}$ of $X$ with $h_{n} \rightarrow h$ satisfying

$$
x_{n}+t_{n} h_{n} \in S \quad \text { for all } \quad n \in \mathbb{N} \text {. }
$$


This set is obviously a cone containing zero and it is known to be closed and convex. The polar cone of $T^{C}(S ; x)$ is the Clarke normal cone $N^{C}(S ; x)$ of $S$ at $x$, that is,

$$
N^{C}(S ; x):=\left\{x^{\star} \in X^{\star}:\left\langle x^{\star}, h\right\rangle \leq 0, \forall h \in T^{C}(S ; x)\right\} .
$$

If $x \notin S$, by convention $T^{C}(S ; x)$ and $N^{C}(S ; x)$ are empty.

Consider now a nonempty open set $U$ of $X$ and an extended real-valued function $f: U \rightarrow \mathbb{R} \cup\{-\infty,+\infty\}$. The Clarke subdifferential $\partial_{C} f(x)$ of $f$ at $x \in U$ is defined by

$$
\partial_{C} f(x):=\left\{x^{\star} \in X^{\star}:\left(x^{\star},-1\right) \in N^{C}(\operatorname{epi} f ;(x, f(x)))\right\},
$$

where $X \times \mathbb{R}$ is endowed with the usual product structure and epi $f$ is the epigraph of $f$, that is,

$$
\text { epi } f:=\left\{\left(x^{\prime}, r\right) \in X \times \mathbb{R}: x^{\prime} \in U, f\left(x^{\prime}\right) \leq r\right\} .
$$

If $f$ is not finite at $x$, we see that $\partial_{C} f(x)=\emptyset$. When $f$ is Lipschitz near $x \in U$ (in particular finite at $x$ ), one defines its Clarke directional derivative at $x$ in the direction $h \in X$ by

$$
f^{o}(x ; h):=\limsup _{t \downarrow 0, x^{\prime} \rightarrow x} t^{-1}\left(f\left(x^{\prime}+t h\right)-f\left(x^{\prime}\right)\right) .
$$

Recall that under such a Lipschitz hypothesis, one has

$$
f^{o}(x ; h)=\max \left\{\left\langle x^{\star}, h\right\rangle: x^{\star} \in \partial_{C} f(x)\right\} \quad \text { for all } h \in X,
$$

that is, $f^{o}(x ; \cdot)$ is the support function of $\partial_{C} f(x)$.

For $x \in S$, one defines $N^{F}(S ; x)$, the Fréchet normal cone of $S$ at $x$ by

$$
N^{F}(S ; x):=\left\{x^{\star} \in X^{\star}: \limsup _{S \ni x^{\prime} \rightarrow x} \frac{\left\langle x^{\star}, x^{\prime}-x\right\rangle}{\left\|x^{\prime}-x\right\|} \leq 0\right\} .
$$

For any $x \in X \backslash S$, one sets again $N^{F}(S ; x)=\emptyset$. Then, the Fréchet subdifferential $\partial_{F} f(x)$ of $f$ at a point $x$ in the open set $U$ is defined by

$$
\partial_{F} f(x):=\left\{x^{\star} \in X^{\star}:\left(x^{\star},-1\right) \in N^{F}(\operatorname{epi} f ;(x, f(x)))\right\},
$$

so $\partial_{F} f(x)=\emptyset$ whenever $f$ is not finite at $x$. If $|f(x)|<+\infty$, one can show that for any $x^{\star} \in X^{\star}, x^{\star} \in \partial_{F} f(x)$ if and only if for any real $\varepsilon>0$, there exists a real $\eta>0$ such that

$$
\left\langle x^{\star}, x^{\prime}-x\right\rangle \leq f\left(x^{\prime}\right)-f(x)+\varepsilon\left\|x^{\prime}-x\right\| \quad \text { for all } x^{\prime} \in B(x, \eta) .
$$

For the Fréchet and Clarke subdifferentials of $d_{S}$ at $x \in S$ one has

$$
\partial_{F} d_{S}(x)=N^{F}(S ; x) \cap \mathbb{B}_{X^{\star}} \quad \text { and } \quad \partial_{C} d_{S}(x) \subset N^{C}(S ; x) \cap \mathbb{B}_{X^{\star}} .
$$

Let $F: X \rightrightarrows Y$ be a multimapping from $X$ to another Banach space $Y,(\bar{x}, \bar{y}) \in$ gph $F:=$ $\{(x, y) \in X \times Y: y \in F(x)\}$. One says that $F$ is metrically regular at $\bar{x}$ for $\bar{y}$ (or around $(\bar{x}, \bar{y})$ ) whenever there exist a real $\gamma \geq 0$ and neighborhoods $U$ and $V$ of $\bar{x}$ and $\bar{y}$ respectively such that

$$
d\left(x, F^{-1}(y)\right) \leq \gamma d(y, F(x)) \text { for all }(x, y) \in U \times V .
$$

The modulus of metric regularity $\operatorname{reg}[F](\bar{x} \mid \bar{y})$ of $F$ at $\bar{x}$ for $\bar{y}$ is defined as the infimum of all $\gamma \in[0,+\infty[$ for which there are neighborhoods $U$ and $V$ of $\bar{x}$ and $\bar{y}$ such that the latter inequality is fulfilled.

For more details, we refer the reader to the books $[11,13,26,36]$. 


\section{Prox-Regularity AND $\omega(\cdot)$-NORMal Regularity CONCEPTS}

In this section, $\mathcal{H}$ is a Hilbert space, $S$ is a nonempty subset of $\mathcal{H}$ and $r$ is an extended real of $] 0,+\infty]$. We will use the classical convention $\frac{1}{r}=0$ whenever $r=+\infty$ and we denote by $U_{r}(S)$ the open $r$-enlargement of the set $S$, that is,

$$
U_{r}(S):=\left\{x \in \mathcal{H}: d_{S}(x)<r\right\} .
$$

The set $S$ is said [31] to be $r$-prox-regular (or uniformly prox-regular with constant $r$ ) whenever it is closed in $\mathcal{H}$ and for any $x \in S$ and any nonzero $v \in \mathcal{H}$ such that $x \in \operatorname{Proj}_{S}(x+v)$, one has $x \in \operatorname{Proj}_{S}\left(x+t \frac{v}{\|v\|}\right)$ for every non-negative real $t \leq r$. A closed subset $S$ in $\mathcal{H}$ is known to be $r$-prox-regular if and only if the mapping $P_{S}: U_{r}(S) \rightarrow \mathcal{H}$ is well defined and norm-to-norm continuous. Obviously, $S$ is $r^{\prime}$-prox-regular for any $\left.\left.r^{\prime} \in\right] 0, r\right]$ whenever it is $r$-prox-regular. It is well-known (see, e.g., [12]) that the set $S$ is $r$-prox-regular if and only if for all $x, x^{\prime} \in S$, for all $v \in N^{C}(S ; x)$ (or $N^{F}(S ; x)$ ),

$$
\left\langle v, x^{\prime}-x\right\rangle \leq \frac{\|v\|}{2 r}\left\|x^{\prime}-x\right\|^{2},
$$

or equivalently (4.1) holds true for any $x, x^{\prime} \in S$ with $N^{C}(S ; x) \neq\{0\}$ and $v \in N^{C}(S ; x)$.

For $\bar{x} \in S$, one says that $S$ is prox-regular at $\bar{x}$ whenever it is closed near $\bar{x}$ and there is a neighborhood $U$ of $\bar{x}$ such that $P_{S}: U \rightarrow \mathcal{H}$ is well defined and norm-to-norm continuous on $U$. If $S$ is nonempty and closed, one says that it is prox-regular whenever it is prox-regular at any of its points. It has been proved that

$$
N^{F}(S ; \bar{x})=N^{C}(S ; \bar{x}),
$$

whenever $S$ is prox-regular at $\bar{x} \in S$. So, in such a case, we set

$$
N(S ; \bar{x}):=N^{F}(S ; \bar{x})=N^{C}(S ; \bar{x}) .
$$

An important characterization of prox-regularity says that the set $S$ is $r$-prox-regular if and only if (4.1) holds only for all $x, x^{\prime} \in S$ satisfying $\left\|x-x^{\prime}\right\|<2 r$. More precisely:

Proposition 4.1. Let $\mathcal{H}$ be a Hilbert space, $S$ be a nonempty closed subset of $\mathcal{H}$ and $s, t$ be two extended reals in $] 0,+\infty]$. The set $S$ is $\min \{s, t\}$-prox-regular whenever for all $x, x^{\prime} \in S$ with $\left\|x-x^{\prime}\right\|<2 t$ and for all $v \in N^{F}(S ; x) \cap \mathbb{B}_{\mathcal{H}}\left(\right.$ resp., $\left.N^{C}(S ; x) \cap \mathbb{B}_{\mathcal{H}}\right),\left\langle v, x^{\prime}-x\right\rangle \leq \frac{1}{2 s}\left\|x^{\prime}-x\right\|^{2}$.

In addition to prox-regular sets, the analysis in the next sections will involve the new class of normally $\omega(\cdot)$-regular sets.

Definition 4.2. Let $X$ be a Banach space, $S$ be a subset of $X, \omega: \mathbb{R}_{+} \rightarrow \mathbb{R}_{+}$be a function with $\omega(0)=0$, $V$ be a nonempty open subset of $X$. One says that $S$ is normally $\omega(\cdot)$-regular relative to $V$ whenever for all $x, x^{\prime} \in S \cap V$, for all $x^{\star} \in N^{C}(S ; x)$,

$$
\left\langle x^{\star}, x^{\prime}-x\right\rangle \leq\left\|x^{\star}\right\| \omega\left(\left\|x^{\prime}-x\right\|\right) .
$$

From (4.1), we see that a nonempty closed subset $S$ of a Hilbert space $\mathcal{H}$ is $r$-prox-regular for some extended real $r \in] 0,+\infty]$ if and only if it is normally $\omega(\cdot)$-regular relative to $\mathcal{H}$ with $\omega: \mathbb{R}_{+} \rightarrow \mathbb{R}_{+}$defined by

$$
\omega(t)=\frac{t^{2}}{2 r} \quad \text { for all } \quad t \in \mathbb{R}_{+} .
$$

There are many normally $\omega(\cdot)$-regular sets which are not prox-regular. 


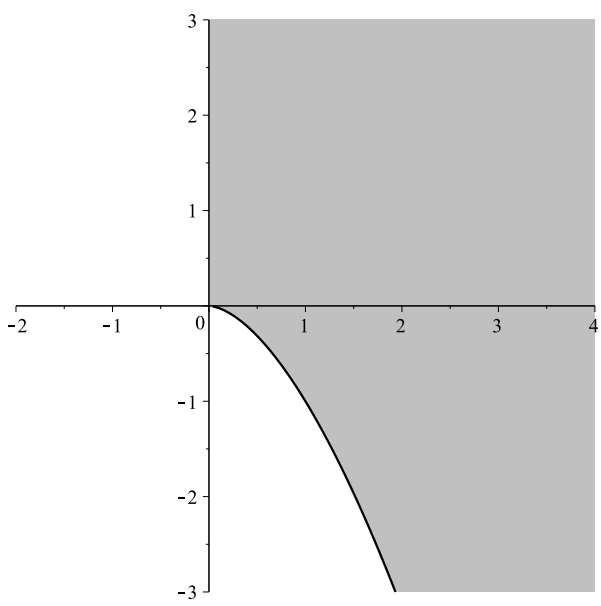

FiguRE 1. A normally $\omega(\cdot)$-regular set non-prox-regular.

Example 4.3. Let $f: \mathbb{R} \rightarrow \mathbb{R} \cup\{+\infty\}$ be the function defined by

$$
f(x):= \begin{cases}-x^{\frac{5}{3}} & \text { if } \quad x \geq 0 \\ +\infty & \text { otherwise }\end{cases}
$$

and set $S:=$ epi $f$. The set $S$ is not uniformly prox-regular but it is normally $\omega(\cdot)$-regular for some function $\omega: \mathbb{R}_{+} \rightarrow \mathbb{R}_{+}$(see, e.g., [29]) defined by

$$
\omega(t):=\alpha t \quad \text { for all } \quad t \in \mathbb{R}_{+},
$$

with $\alpha \in \mathbb{R}_{+}$.

Our developement below will involve multimappings with normally $\omega(\cdot)$-regular graphs. The following result provides sufficient conditions to ensure the normal $\omega(\cdot)$-regularity of gph $M$, for a multimapping $M$ given as a sum of a mapping and a set.

Theorem 4.4. Let $X, Y$ be Banach spaces, $\omega: \mathbb{R}_{+} \rightarrow \mathbb{R}_{+}$be a nondecreasing function with $\omega(0)=0$, $S$ be a normally $\omega(\cdot)$-regular subset of $Y, G: X \rightarrow Y$ be a mapping, and $M(\cdot)=G(\cdot)+S$. Assume that:

(i) the mapping $G$ is differentiable on $X$ and there exists a real $\gamma \geq 0$ such that

$$
\|D G(x)-D G(y)\| \leq \gamma\|x-y\| \quad \text { for all } \quad x, y \in X ;
$$

(ii) there exists a real $K \geq 0$ such that

$$
\left\|y-G(x)-\left(y^{\prime}-G\left(x^{\prime}\right)\right)\right\| \leq K\left\|(x, y)-\left(x^{\prime}, y^{\prime}\right)\right\| \quad \text { for all } \quad \in(x, y),\left(x^{\prime}, y^{\prime}\right) \in \operatorname{gph} M .
$$

Then, the set gph $M$ is normally $\rho(\cdot)$-regular where $\rho: \mathbb{R}_{+} \rightarrow \mathbb{R}_{+}$is defined by

$$
\rho(t):=\omega(K t)+\frac{\gamma t^{2}}{2} \quad \text { for all } \quad t \in \mathbb{R}_{+} .
$$

Proof. Fix any $(x, y),\left(x^{\prime}, y^{\prime}\right) \in$ gph $M$. It is well-known (and not difficult to check) that

$$
N^{C}(\operatorname{gph} M ;(x, y))=\left\{\left(-D G(x)^{\star}\left(y^{\star}\right), y^{\star}\right): y^{\star} \in N(S ; y-G(x))\right\},
$$


where $D G(x)^{\star}$ denotes the adjoint operator of $D G(x)$. Let $\left(u^{\star}, v^{\star}\right) \in N^{C}(\operatorname{gph} M ;(x, y))$. Thanks to the latter equality, we have $v^{\star} \in N(S ; y-G(x))$ and $u^{\star}=-D G(x)^{\star}\left(v^{\star}\right)$, and hence

$$
\left\langle\left(u^{\star}, v^{\star}\right),\left(x^{\prime}, y^{\prime}\right)-(x, y)\right\rangle=\left\langle-v^{\star}, D G(x)\left(x^{\prime}-x\right)\right\rangle+\left\langle v^{\star}, y^{\prime}-y\right\rangle .
$$

It is readily seen that

$$
\left\langle v^{\star}, y^{\prime}-y\right\rangle=\left\langle v^{\star},\left(y^{\prime}-G\left(x^{\prime}\right)\right)-(y-G(x))\right\rangle+\left\langle v^{\star}, G\left(x^{\prime}\right)-G(x)\right\rangle .
$$

Thanks to $(i)$, we also have

$$
\begin{aligned}
\left\langle-v^{\star}, D G(x)\left(x^{\prime}-x\right)\right\rangle= & \left\langle v^{\star}, \int_{0}^{1}\left(D G\left(x+\tau\left(x^{\prime}-x\right)\right)-D G(x)\right)\left(x^{\prime}-x\right) \mathrm{d} \tau\right\rangle \\
& +\left\langle-v^{\star}, G\left(x^{\prime}\right)-G(x)\right\rangle .
\end{aligned}
$$

From (4.3) and the fact that $S$ is $\omega(\cdot)$-normally regular, we deduce

$$
\left\langle v^{\star}, y^{\prime}-y\right\rangle \leq\left\|v^{\star}\right\| \omega\left(\left\|\left(y^{\prime}-G\left(x^{\prime}\right)\right)-(y-G(x))\right\|\right)+\left\langle v^{\star}, G\left(x^{\prime}\right)-G(x)\right\rangle .
$$

Combining (4.2), (4.5), (4.4), (i) and (ii) we obtain

$$
\begin{aligned}
& \left\langle\left(u^{\star}, v^{\star}\right),\left(x^{\prime}, y^{\prime}\right)-(x, y)\right\rangle \\
\leq & \left\|v^{\star}\right\| \omega\left(\left\|\left(y^{\prime}-G\left(x^{\prime}\right)\right)-(y-G(x))\right\|\right)+\left\langle v^{\star}, \int_{0}^{1}\left(D G\left(x+\tau\left(x^{\prime}-x\right)\right)-D G(x)\right)\left(x^{\prime}-x\right) \mathrm{d} \tau\right\rangle \\
\leq & \left\|v^{\star}\right\| \omega\left(K\left\|\left(x^{\prime}, y^{\prime}\right)-(x, y)\right\|\right)+\left\|v^{\star}\right\|\left\|x^{\prime}-x\right\|^{2} \int_{0}^{1} \gamma \tau \mathrm{d} \tau . \\
\leq & \left\|\left(u^{\star}, v^{\star}\right)\right\|\left(\omega\left(K\left\|\left(x^{\prime}, y^{\prime}\right)-(x, y)\right\|\right)+\frac{\gamma}{2}\left\|\left(x^{\prime}, y\right)^{\prime}-(x, y)\right\|^{2}\right) .
\end{aligned}
$$

As a consequence, the set gph $M$ is $\rho(\cdot)$-normally regular.

The latter theorem in the setting of prox-regular sets is of great interest.

Corollary 4.5. Let $\mathcal{H}, \mathcal{H}^{\prime}$ be Hilbert spaces, $S$ be an r-prox-regular subset of $\mathcal{H}^{\prime}$ for some extended real $r \in$ ]0, $+\infty], G: \mathcal{H} \rightarrow \mathcal{H}^{\prime}$ be a mapping, and $M(\cdot)=G(\cdot)+S$. Assume that the conditions (i) and (ii) in Theorem 4.4 are satisfied. Then, the set gph $M$ is $\frac{1}{\frac{K^{2}}{r}+\gamma}$-prox-regular.

Remark 4.6. With $S=\{0\}$, the latter corollary says in particular that a differentiable mapping $f: \mathcal{H} \rightarrow \mathcal{H}^{\prime}$ from a Hilbert space $\mathcal{H}$ to a Hilbert space $\mathcal{H}^{\prime}$ with $\gamma$-Lipschitz continuous derivative for some real $\gamma \geq 0$ has a $\frac{1}{\gamma}$-prox-regular graph. It is worth pointing out that this can also be seen as a consequence of ([2], Thm. 3.3).

\section{Metric REgularity via PROX-REgularity/NORMAL $\omega(\cdot)$-REgUlarity}

Let $M: X \rightrightarrows Y$ be a multimapping between two Banach spaces with closed graph and let $(\bar{x}, \bar{y}) \in \operatorname{gph} M$. Recall that $M$ is said to be graph-convex whenever gph $M$ is convex. It is well-known (and not difficult to prove) that $M$ is graph-convex if and only if for all $x, y \in X$, for all $\lambda \in] 0,1[$,

$$
\lambda M(x)+(1-\lambda) M(y) \subset M(\lambda x+(1-\lambda) y) .
$$

For such a multimapping $M$, Robinson [35] and Ursescu [41] proved independently in 1975-1976 that $M$ is metrically regular at $\bar{x}$ for $\bar{y}$ if and only if $\bar{y} \in$ Core $M(X)$, where

$$
\text { Core } M(X)=\left\{y \in Y: \forall y^{\prime} \in Y, \exists r>0, y+t y^{\prime} \in M(X), \forall t \in[-r, r]\right\} .
$$


This result can be viewed as an extension of the classical Banach open mapping theorem. More precisely, whenever $\bar{y} \in$ Core $M(X)$, there is some real $c>0$ such that

$$
d\left(x, M^{-1}(y)\right) \leq(c-\|y-\bar{y}\|)^{-1}(1+\|x-\bar{x}\|) d(y, M(x)) \quad \text { for all } x \in X, y \in B(\bar{y}, c) .
$$

For the extension of such an inequality to more general multimappings, under a point-based open condition $\bar{y}+\beta \mathbb{U}_{Y} \subset M\left(\bar{x}+\alpha \mathbb{B}_{X}\right)$, the class of paraconvex multimappings is a first appropriate one (see, e.g., $\left.[17,18]\right)$. Recall that $M$ is said to be $(\gamma, C)$-paraconvex ([37]) for a real $\gamma>0$ and a real $C \geq 0$ whenever for all $x_{1}, x_{2} \in X$, for all $t \in[0,1]$,

$$
t M\left(x_{1}\right)+(1-t) M\left(x_{2}\right) \subset M\left(t x_{1}+(1-t) x_{2}\right)+C \min (t, 1-t)\left\|x_{1}-x_{2}\right\|^{\gamma} \mathbb{B}_{Y} .
$$

In [17], Huang and Li established that if $M^{-1}$ is paraconvex, then $M$ is metrically regular at $\bar{x}$ for $\bar{y}$ whenever $\bar{y}+\beta \mathbb{U}_{Y} \subset M\left(\bar{x}+\alpha \mathbb{B}_{X}\right)$ for some reals $\left.\alpha, \beta \in\right] 0,+\infty[$. In fact, under the latter inclusion, from ([17], Thm. 2.2) we have the following estimate for any $\left.\eta, \eta^{\prime} \in\right] 0,+\infty\left[\right.$ with $\eta+\eta^{\prime}=\beta$,

$$
d\left(x, M^{-1}(y)\right) \leq \eta^{-1}\left(\alpha+C \eta^{\gamma}+\|x-\bar{x}\|\right) d(y, M(x))
$$

for all $x \in X$ and for all $y \in \bar{y}+\eta^{\prime} \mathbb{U}_{Y}$, where $\left.\gamma, C \in\right] 0,+\infty\left[\right.$ are such that $M^{-1}$ is $(\gamma, C)$-paraconvex. We also refer to Jourani ([18]) for another previous result of this type.

In [47], Zheng and Ng showed that prox-regularity is also a suitable class for extension of Robinson-Ursescu theorem. For $X$ and $Y$ Hilbert spaces and gph $M(r, \delta)$-prox-regular at $(\bar{x}, \bar{y})$ for some reals $r, \delta>0$, that is

$$
\left\langle\left(x^{\star}, y^{\star}\right),(u, v)-(x, y)\right\rangle \leq \frac{1}{2 r}\|(u, v)-(x, y)\|^{2},
$$

for all $(u, v),(x, y) \in \operatorname{gph} M \cap(B(\bar{x}, \delta) \times B(\bar{y}, \delta))$, for all $\left(x^{\star}, y^{\star}\right) \in N^{C}(\operatorname{gph} M ;(x, y)) \cap\left(\mathbb{B}_{X} \times \mathbb{B}_{Y}\right)$, they proved that $M$ is metrically regular at $\bar{x}$ for $\bar{y}$ whenever

$$
\bar{y}+\beta \mathbb{U}_{Y} \subset M\left(\bar{x}+\alpha \mathbb{U}_{X}\right)
$$

for some reals $\alpha \in] 0, \frac{\delta}{3}[, \beta \in] 0, \delta\left[\right.$ satisfying $\beta>\frac{4 \alpha^{2}+\beta^{2}}{2 r}$.

More recently, X.Y. Zheng and Q.H. He developed a Robinson-Ursescu type theorem type for multimappings with $(\sigma, \delta)$-subsmooth graph. The $(\sigma, \delta)$-subsmoothness of sets which is a quantified version of subsmooth sets introduced by Aussel, Daniilidis and Thibault [5] is defined as follows.

Definition 5.1 ([44]). Let $X$ be a Banach space, $S$ be a subset of $X, \bar{x} \in S, \sigma, \delta \in] 0,+\infty[$. One says that $S$ is $(\sigma, \delta)$-subsmooth at $\bar{x}$ if for all $x, x^{\prime} \in S \cap B(\bar{x}, \delta)$, for all $x^{\star} \in N^{C}(S ; x)$,

$$
\left\langle x^{\star}, x^{\prime}-x\right\rangle \leq \sigma\left\|x^{\star}\right\|\left\|x^{\prime}-x\right\| .
$$

Note that a closed set $S \subset X$ which is $(\sigma, \delta)$-subsmooth at $\bar{x} \in S$ with $\sigma, \delta \in] 0,+\infty[$, is obviously normally $\omega(\cdot)$-regular relative to $B(\bar{x}, \delta)$ for $\omega: \mathbb{R}_{+} \rightarrow \mathbb{R}_{+}$defined by

$$
\omega(t)=\sigma t \quad \text { for all } t \in \mathbb{R}_{+} .
$$

For a multimapping $M$ between the Banach spaces $X$ and $Y$, the metric regularity at $\bar{x}$ for $\bar{y}$ is obtained in [44] with $\alpha, \beta \in] 0, \delta$ [ satisfying

$$
\frac{1}{\sigma}-1>\frac{2 \alpha}{\beta}
$$

for any $(\sigma, \delta) \in] 0,1[\times] 0,+\infty[$ such that gph $M$ is $(\sigma, \delta)$-subsmooth at $(\bar{x}, \bar{y})$. 


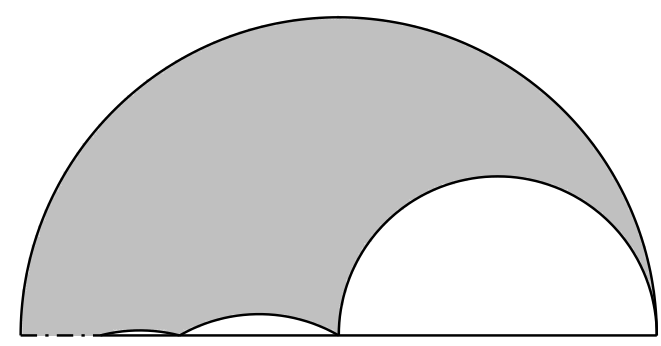

FiguRE 2. The graph of $M$.

In order to establish our results concerned with uniform prox-regularity of sets, we need to develop a verifiable sufficient condition ensuring a kind of uniform metric regularity. Given a multimapping $M: X \rightrightarrows Y$ and a set $Q \subset$ gph $M$, we want to find some real $\gamma \geq 0$ such that for any $(\bar{x}, \bar{y}) \in Q$,

$$
d\left(x, M^{-1}(y)\right) \leq \gamma d(y, M(x)),
$$

for all $(x, y)$ near $(\bar{x}, \bar{y})$. We therefore need to require a uniform point-based condition as well as the uniform prox-regularity of gph $M$. This leads to give first some generalities about multimappings with prox-regular graph.

It is worth pointing out that the graph convexity of $M$ is equivalent to the graph convexity of $M^{-1}$ (resp., $-M$ ). The following result, which states that a similar preservation still holds for the prox-regularity, will be needed in the developement below. We omit the proof since it can be established in a straightforward way.

Proposition 5.2. Let $\mathcal{H}, \mathcal{H}^{\prime}$ be Hilbert spaces, $M: \mathcal{H} \rightrightarrows \mathcal{H}^{\prime}$ be a multimapping, $\left.\left.r \in\right] 0,+\infty\right]$ be an extended real. Then, gph $M$ is r-prox-regular if and only if gph $M^{-1}$ (resp., $\operatorname{gph}(-M)$ ) is r-prox-regular.

It is also known and easy to see that the property of graph-convexity of $M$ entails that $M(C):=\bigcup_{x \in C} M(x)$ is convex for any convex set $C \subset X$ (in particular $\operatorname{ker} M:=M^{-1}(0)$ is convex) as well as Dom $M:=\{x \in X$ : $M(x) \neq \emptyset\})$. The aim of Examples 5.3 and 7.13 below is to show that such preservations fail for a multimapping with prox-regular graph.

Example 5.3. Set $R=\frac{1}{2}$. Consider for each $n \in \mathbb{N}$, the closed ball $D_{n}$ of $\mathbb{R}^{2}$ with radius $r=\frac{1}{4}$ (independent of $n)$ with $\left(0, \frac{1}{2^{n-1}}\right)$ and $\left(0, \frac{1}{2^{n}}\right)$ on its boundary and whose abscissa of its center is nonpositive. As in $[2,6]$, we define

$$
Q=\left\{(x, y) \in \mathbb{R}^{2}: x \geq 0, x^{2}+\left(y-\frac{1}{2}\right)^{2} \leq R^{2}\right\} \backslash \bigcup_{n \in \mathbb{N}} \operatorname{int} D_{n} .
$$

The set $Q$ is uniformly prox-regular. The multimapping $M: \mathbb{R} \rightrightarrows \mathbb{R}$ with gph $M:=Q$ has an image

$$
M(0)=\{0,1\} \cup\left\{\frac{1}{2^{n}}: n \in \mathbb{N}\right\}
$$

not (uniformly) prox-regular. On the other hand, according to Proposition 5.2, with $N:=M^{-1}$ the set gph $N$ is uniformly prox-regular and $N^{-1}(0)=M(0)$ is not uniformly prox-regular.

To obtain prox-regularity thickness in the results below, we will need to quantify the modulus of such a metric regularity, i.e., to give an estimate of the real $\gamma$ in (5.1). Our theorem requires the existence of three reals $\alpha, \beta, \rho>0$ such that the uniform openness condition

$$
\bar{y}+\beta \mathbb{U}_{Y} \subset M\left(\bar{x}+\alpha \mathbb{B}_{X}\right) \quad \text { for all }(\bar{x}, \bar{y}) \in Q
$$


holds as well as the inequality

$$
\beta>\frac{3 \alpha}{\rho}+\left(1+\frac{1}{\rho}\right) \omega\left(\sqrt{4 \alpha^{2}+\left(\beta-\frac{\alpha}{\rho}\right)^{2}}\right) .
$$

Fix three reals $\alpha, \beta, \rho>0$. First, let us look at the case when gph $M$ is $r$-prox-regular for some $r \in] 0,+\infty[$, i.e., gph $M$ is normally $\omega(\cdot)$-regular relative to $X \times Y$ (here $X$ and $Y$ are assumed to be Hilbert spaces) with $\omega: \mathbb{R}_{+} \rightarrow \mathbb{R}_{+}$defined by

$$
\omega(t)=\frac{t^{2}}{2 r} \quad \text { for all } t \in \mathbb{R}_{+}
$$

If $\alpha<\frac{r}{2}$ and $|\beta-r|<\sqrt{r^{2}-4 \alpha^{2}}$, then we have

$$
\frac{4 \alpha^{2}+\beta^{2}}{2 r}<\beta
$$

hence there is a real $\rho>0$ such that

$$
\beta>\frac{3 \alpha}{\rho}+\frac{1}{2 r}\left(1+\frac{1}{\rho}\right)\left[4 \alpha^{2}+\left(\beta-\frac{\alpha}{\rho}\right)^{2}\right] .
$$

Now, assume that gph $M$ is $(\sigma, \delta)$-subsmooth at $(\bar{x}, \bar{y}) \in \operatorname{gph} M$ for any reals $\sigma \in] 0,1[$ and $\delta \in] 0,+\infty[$, i.e., gph $M$ is normally $\omega(\cdot)$-regular relative to $B((\bar{x}, \bar{y}), \delta)$ with $\omega: \mathbb{R}_{+} \rightarrow \mathbb{R}_{+}$defined by

$$
\omega(t)=\sigma t \quad \text { for all } t \in \mathbb{R}_{+} .
$$

If $\alpha<\frac{\beta}{2 \sigma} \sqrt{1-\sigma^{2}}$, then we have

$$
\sigma \sqrt{4 \alpha^{2}+\beta^{2}}<\beta
$$

Thus, one can find a real $\rho>0$ such that

$$
\beta>\frac{3 \alpha}{\rho}+\sigma\left(1+\frac{1}{\rho}\right) \sqrt{4 \alpha^{2}+\left(\beta-\frac{\alpha}{\rho}\right)^{2}} .
$$

Now, we can state the theorem on metric regularity of multimappings with normally $\omega(\cdot)$-regular graph. It is an adaptation of ideas of Zheng and $\mathrm{Ng}([47])$.

Theorem 5.4. Let $X, Y$ be Banach spaces, $M: X \rightrightarrows Y$ be a multimapping with closed graph, $Q$ be a nonempty subset of gph $M, \omega: \mathbb{R}_{+} \rightarrow \mathbb{R}_{+}$an upper semicontinuous nondecreasing function with $\omega(0)=0$. Assume that:

(i) there exist $\alpha, \beta, \rho \in] 0,+\infty[$ with

$$
\beta>\frac{3 \alpha}{\rho}+\left(1+\frac{1}{\rho}\right) \omega\left(\sqrt{4 \alpha^{2}+\left(\beta-\frac{\alpha}{\rho}\right)^{2}}\right)
$$

such that for all $(\bar{x}, \bar{y}) \in Q$,

$$
\bar{y}+\beta \mathbb{U}_{Y} \subset M\left(\bar{x}+\alpha \mathbb{B}_{X}\right) ;
$$

(ii) the set gph $M$ is normally $\omega(\cdot)$-regular relative to $\bigcup_{(a, b) \in Q} B\left((a, b), \sqrt{\alpha^{2}+\beta^{2}}\right)$. 
Then, there exists a real $\gamma \in[0, \rho[$ such that for every $(\bar{x}, \bar{y}) \in Q$, there exists a real $\delta>0$ satisfying for all $x \in B(\bar{x}, \delta)$, for all $y \in B(\bar{y}, \delta)$,

$$
d\left(x, M^{-1}(y)\right) \leq \gamma d(y, M(x))
$$

In particular, one has $\sup _{(x, y) \in Q} \operatorname{Reg}[M](x \mid y) \leq \rho$.

Proof. Set $V=\bigcup_{(a, b) \in Q} B\left((a, b), \sqrt{\alpha^{2}+\beta^{2}}\right)$. By contradiction, assume that for each $\gamma \in[0, \rho[$, there is $(\bar{x}, \bar{y}) \in Q$ such that for each real $\delta>0$, there are $x \in B(\bar{x}, \delta), y \in B(\bar{y}, \delta)$ satisfying

$$
d\left(x, M^{-1}(y)\right)>\gamma d(y, M(x)) .
$$

Let $\left(\varepsilon_{n}\right)_{n \in \mathbb{N}}$ be a nonincreasing sequence of $] 0, \rho\left[\right.$ with $\varepsilon_{n} \downarrow 0$. For each $n \in \mathbb{N}$, set

$$
\theta_{n}=\frac{1}{\rho-\varepsilon_{n}}\left(\alpha+\frac{1}{n}\right)+\frac{1}{n}
$$

Obviously, $\left(\theta_{n}\right)_{n \in \mathbb{N}}$ is a nonincreasing sequence which satisfies $\theta_{n} \rightarrow \frac{\alpha}{\rho}$. Since we have $\beta>\frac{\alpha}{\rho}$, there is $n_{0} \in \mathbb{N}$ such that for all integer $n \geq n_{0}$,

$$
\theta_{n}<\beta
$$

Choose $N \in \mathbb{N}$ with $N \geq n_{0}$ such that for all integer $n \geq N$,

$$
\left(\alpha+\frac{2}{n}\right)^{2}+\theta_{n}^{2}<\alpha^{2}+\beta^{2}
$$

Fix any integer $n \geq N$. There are $\left(\bar{x}_{n}, \bar{y}_{n}\right) \in Q, x_{n} \in X, y_{n} \in Y$ with

$$
\left\|\bar{x}_{n}-x_{n}\right\|<\min \left\{\beta, \frac{1}{\rho-\varepsilon_{n}}, \frac{1}{n}\right\} \quad \text { and } \quad\left\|\bar{y}_{n}-y_{n}\right\|<\min \left\{\beta, \frac{1}{\rho-\varepsilon_{n}}, \frac{1}{n}\right\}
$$

and

$$
d\left(x_{n}, M^{-1}\left(y_{n}\right)\right)>\left(\rho-\varepsilon_{n}\right) d\left(y_{n}, M\left(x_{n}\right)\right) .
$$

According to ([46], Lem. 3.1), there is $\left(u_{n}, v_{n}\right) \in \operatorname{gph} M$ such that

$$
\begin{gathered}
\left\|u_{n}-x_{n}\right\|<d\left(x_{n}, M^{-1}\left(y_{n}\right)\right), \\
0<\left\|v_{n}-y_{n}\right\|<\frac{d\left(x_{n}, M^{-1}\left(y_{n}\right)\right)}{\rho-\varepsilon_{n}}
\end{gathered}
$$

and

$$
(0,0) \in\{0\} \times J\left(v_{n}-y_{n}\right)+\frac{1}{\rho-\varepsilon_{n}}\left(\mathbb{B}_{X^{\star}} \times \varepsilon_{n} \mathbb{B}_{Y^{\star}}\right)+N^{C}\left(\operatorname{gph} M ;\left(u_{n}, v_{n}\right)\right),
$$

where $J=\partial(\|\cdot\|)$ is the subdifferential in the sense of convex analysis of $\|\cdot\|$. Since $v_{n}-y_{n} \neq 0$, there is some $z_{n}^{\star} \in Y^{\star}$ such that

$$
\left\|z_{n}^{\star}\right\|=1 \quad \text { and } \quad\left\langle z_{n}^{\star}, v_{n}-y_{n}\right\rangle=\left\|v_{n}-y_{n}\right\|
$$

and there is some $\left(x_{n}^{\star}, y_{n}^{\star}\right) \in N^{C}\left(\operatorname{gph} M ;\left(u_{n}, v_{n}\right)\right)$ satisfying

$$
\left(x_{n}^{\star}, y_{n}^{\star}\right) \in\left(0,-z_{n}^{\star}\right)+\frac{1}{\rho-\varepsilon_{n}}\left(\mathbb{B}_{X^{\star}} \times \varepsilon_{n} \mathbb{B}_{Y^{\star}}\right) .
$$


It follows that

$$
\left\|x_{n}^{\star}\right\| \leq \frac{1}{\rho-\varepsilon_{n}}
$$

and

$$
y_{n}^{\star} \in-z_{n}^{\star}+\frac{\varepsilon_{n}}{\rho-\varepsilon_{n}} \mathbb{B}_{Y^{\star}} .
$$

Since $\left(\bar{x}_{n}, \bar{y}_{n}\right) \in Q$, we can apply our assumption to obtain

$$
B\left(\bar{y}_{n}, \beta\right) \subset M\left(B\left[\bar{x}_{n}, \alpha\right]\right) .
$$

Using (5.3), we know that $y_{n} \in B\left(\bar{y}_{n}, \beta\right)$, so there is $z_{n} \in B\left[\bar{x}_{n}, \alpha\right]$ such that

$$
y_{n} \in M\left(z_{n}\right) \text {. }
$$

In particular, we have

$$
z_{n} \in M^{-1}\left(y_{n}\right) \cap B\left[\bar{x}_{n}, \alpha\right] .
$$

Keeping in mind (5.3), the latter inclusion entails

$$
d\left(x_{n}, M^{-1}\left(y_{n}\right)\right) \leq\left\|x_{n}-z_{n}\right\| \leq\left\|x_{n}-\bar{x}_{n}\right\|+\left\|\bar{x}_{n}-z_{n}\right\| \leq \frac{1}{n}+\alpha .
$$

From (5.4), (5.3) and the latter inequality, it ensues that

$$
\left\|u_{n}-\bar{x}_{n}\right\| \leq\left\|u_{n}-x_{n}\right\|+\left\|x_{n}-\bar{x}_{n}\right\|<d\left(x_{n}, M^{-1}\left(y_{n}\right)\right)+\frac{1}{n} \leq \frac{2}{n}+\alpha .
$$

Using (5.4), (5.5) and (5.10), we obtain

$$
\begin{aligned}
\left\|v_{n}-\bar{y}_{n}\right\| & \leq\left\|v_{n}-y_{n}\right\|+\left\|y_{n}-\bar{y}_{n}\right\|<\frac{d\left(x_{n}, M^{-1}\left(y_{n}\right)\right)}{\rho-\varepsilon_{n}}+\frac{1}{n} \\
& \leq \frac{\frac{1}{n}+\alpha}{\rho-\varepsilon_{n}}+\frac{1}{n}=\theta_{n} .
\end{aligned}
$$

Set

$$
\zeta_{n}=\left(\beta-\theta_{n}\right) \frac{v_{n}-y_{n}}{\left\|v_{n}-y_{n}\right\|} .
$$

Thanks to the definition of $\zeta_{n}$, the choice of $n$ and the inequality (5.12), we can check that

$$
v_{n}-\zeta_{n} \in B\left(\bar{y}_{n}, \beta\right)
$$

From the above inclusion and the inclusion (5.9), we get

$$
v_{n}-\zeta_{n} \in M\left(B\left[\bar{x}_{n}, \alpha\right]\right) .
$$

Let $w_{n} \in B\left[\bar{x}_{n}, \alpha\right]$ be such that

$$
v_{n}-\zeta_{n} \in M\left(w_{n}\right)
$$

By (5.11), let us observe that

$$
\left\|w_{n}-u_{n}\right\| \leq\left\|w_{n}-\bar{x}_{n}\right\|+\left\|\bar{x}_{n}-u_{n}\right\|<2 \alpha+\frac{2}{n}
$$


Endow $X \times Y$ with the 2-norm. We can see that

$$
\begin{aligned}
\left\|\left(w_{n}, v_{n}-\zeta_{n}\right)-\left(\bar{x}_{n}, \bar{y}_{n}\right)\right\|^{2} & =\left\|w_{n}-\bar{x}_{n}\right\|^{2}+\left\|v_{n}-\zeta_{n}-\bar{y}_{n}\right\|^{2} \\
& <\alpha^{2}+\beta^{2} .
\end{aligned}
$$

According to (5.2), (5.11) and (5.12), we get

$$
\begin{aligned}
\left\|\left(u_{n}, v_{n}\right)-\left(\bar{x}_{n}, \bar{y}_{n}\right)\right\|^{2} & =\left\|u_{n}-\bar{x}_{n}\right\|^{2}+\left\|v_{n}-\bar{y}_{n}\right\|^{2} \\
& \leq\left(\alpha+\frac{2}{n}\right)^{2}+\theta_{n}^{2} \\
& <\alpha^{2}+\beta^{2} .
\end{aligned}
$$

In particular, we have the inclusions

$$
\left(w_{n}, v_{n}-\zeta_{n}\right),\left(u_{n}, v_{n}\right) \in V .
$$

Further, from the normal $\omega(\cdot)$-regularity of gph $M$ relative to $V$, we have

$$
\left\langle\left(x_{n}^{\star}, y_{n}^{\star}\right),\left(w_{n}, v_{n}-\zeta_{n}\right)-\left(u_{n}, v_{n}\right)\right\rangle \leq\left\|\left(x_{n}^{\star}, y_{n}^{\star}\right)\right\| \omega\left(\left\|\left(w_{n}, v_{n}-\zeta_{n}\right)-\left(u_{n}, v_{n}\right)\right\|\right),
$$

and from (5.7) along with (5.8), it can be easily checked that

$$
\left\|\left(x_{n}^{\star}, y_{n}^{\star}\right)\right\| \leq\left\|x_{n}^{\star}\right\|+\left\|y_{n}^{\star}\right\| \leq 1+\frac{1+\varepsilon_{n}}{\rho-\varepsilon_{n}} .
$$

Hence, it follows

$$
\left\langle x_{n}^{\star}, w_{n}-u_{n}\right\rangle \leq\left\langle y_{n}^{\star}, \zeta_{n}\right\rangle+\left(1+\frac{1+\varepsilon_{n}}{\rho-\varepsilon_{n}}\right) \omega\left(\left\|\left(w_{n}-u_{n},-\zeta_{n}\right)\right\|\right) .
$$

From (5.7) again we also have

$$
\begin{aligned}
\left\langle x_{n}^{\star}, w_{n}-u_{n}\right\rangle & \geq-\left\|x_{n}^{\star}\right\|\left\|w_{n}-u_{n}\right\| \\
& \geq-\frac{1}{\rho-\varepsilon_{n}}\left(2 \alpha+\frac{2}{n}\right) .
\end{aligned}
$$

Note by (5.8), (5.6) and the definition of $\zeta_{n}$ that there is $b_{n}^{\star} \in \mathbb{B}_{Y^{\star}}$ such that

$$
\left\langle y_{n}^{\star}, \zeta_{n}\right\rangle=\left\langle-z_{n}^{\star}+\frac{\varepsilon_{n}}{\rho-\varepsilon_{n}} b_{n}^{\star}, \zeta_{n}\right\rangle \leq \theta_{n}-\beta+\frac{\varepsilon_{n}}{\rho-\varepsilon_{n}}\left(\beta-\theta_{n}\right) .
$$

Note also that (thanks to the nondecreasing property of $\omega$ )

$$
\omega\left(\left\|\left(w_{n}-u_{n},-\zeta_{n}\right)\right\|\right) \leq \omega\left(\left[\left(2 \alpha+\frac{2}{n}\right)^{2}+\left(\beta-\theta_{n}\right)^{2}\right]^{\frac{1}{2}}\right)
$$

Coming back to (5.13), we deduce

$$
-\frac{1}{\rho-\varepsilon_{n}}\left(2 \alpha+\frac{2}{n}\right) \leq-\beta+\theta_{n}+\frac{\varepsilon_{n}}{\rho-\varepsilon_{n}}\left(\beta-\theta_{n}\right)+\left(1+\frac{1+\varepsilon_{n}}{\rho-\varepsilon_{n}}\right) \omega\left(\left[\left(2 \alpha+\frac{2}{n}\right)^{2}+\left(\beta-\theta_{n}\right)^{2}\right]^{\frac{1}{2}}\right) .
$$

Passing to the limsup and using the upper semicontinuity of $\omega$, we get

$$
-\frac{2 \alpha}{\rho} \leq-\beta+\frac{\alpha}{\rho}+\left(1+\frac{1}{\rho}\right) \omega\left(\left(4 \alpha^{2}+\left(\beta-\frac{\alpha}{\rho}\right)^{2}\right)^{\frac{1}{2}}\right)
$$

and this cannot hold true. 


\section{Prox-Regularity of the solution mappings to Generalized equations}

Given a Hilbert space $\mathcal{H}$, a time interval $[0, T]$ with $T>0$, an $r$-prox-regular moving set $C:[0, T] \rightrightarrows \mathcal{H}$ for some $r \in] 0,+\infty]$ and an initial condition $u_{0} \in C\left(T_{0}\right)$, one can consider the problem of finding all the absolutely continuous mappings $u:[0, T] \rightarrow \mathcal{H}$ satisfying

$$
(\mathcal{P})\left\{\begin{array}{l}
-\dot{u}(t) \in N(C(t) ; u(t)) \quad \text { a.e. } t \in[0, T], \\
u(t) \in C(t) \quad \text { for all } t \in[0, T] \\
u(0)=u_{0} .
\end{array}\right.
$$

Such a differential inclusion has been introduced and extensively developped by Moreau [27] in the early 70s in the convex setting (that is, $r=+\infty$ ). For a closed convex set $C(t)$ moving in an absolutely continuous way, Moreau shows that $(\mathcal{P})$ is well posed, in the sense of existence and uniqueness of a solution. The so-called Moreau sweeping process is known to play a crucial role in many applications of mathematics as, for example, in the study of crowd motion ([24]). Removing the convexity assumption on $C(\cdot)$ has been and still remains a very well-active area of research involving suitable classes of sets adapted to this kind of problem, for instance: prox-regular sets, subsmooth sets and $\alpha$-far sets (see, e.g., [19]). It has been well-recognized that (uniform) prox-regularity is the general condition to go beyond the convexity and under which the problem $(\mathcal{P})$ has a unique solution (see, e.g., [12] and the references therein). It is then of a great interest to develop general results of preservation for prox-regular sets. A first step has been achieved in that direction in [2] (see also Vial [43] and Venel [42]) with the case of a moving set described by finitely many inequality/equality constraints,

$$
C(t):=\left\{x \in \mathcal{H}: g_{1}(t, x) \leq 0, \ldots, g_{m}(t, x) \leq 0, g_{m+1}(t, x)=0, \ldots, g_{m+n}(t, x)=0\right\},
$$

where $g_{1}, \ldots, g_{m+n}$ are real-valued functions defined on $\mathcal{H}$. In order to get other shapes of prox-regular sets, one can naturally consider the case where the moving set $C(t)$ is the solution set of a time-dependent generalized equation, that is,

$$
C(t):=\{x \in \mathcal{H}: 0 \in f(t, x)+F(t, x)\},
$$

where $f:[0, T] \times \mathcal{H} \rightarrow \mathcal{H}$ is a mapping and $F:[0, T] \times \mathcal{H} \rightrightarrows \mathcal{H}$ is a multimapping. It is worth pointing out that with $f=\left(g_{1}, \ldots, g_{m+n}\right)$ and $F(x, t)=\mathbb{R}_{-}^{m} \times\left\{0_{\mathbb{R}^{n}}\right\}$ the set given by (6.2) is reduced to (6.1). The term generalized equation, introduced by Robinson in the autonomous case in [32,33], refers to the fact that (6.2) reduces to the ordinary equation $f(t, x)=0$ whenever $F \equiv 0$. An important class of generalized equations is given by the variational inequalities, the case where $F(\cdot)=N(C ; \cdot)$ for some closed convex subset $C$ of $X$, i.e.,

$$
f(x)+N(C ; x) \ni 0 .
$$

It is well established that the concept of generalized equations has a fundamental role in analysis and it possesses many applications in mathematical programming, complementarity problems, economics; for more details, we refer the reader to $[13,34]$ and the references therein. Note that sweeping process with moving set $C(t)$ in $(6.2)$ provides, in addition to Section 2, another area of applications of prox-regularity of solution set of generalized equations.

Given two multimappings $F_{1}, F_{2}: \mathcal{H} \rightrightarrows \mathcal{H}^{\prime}$ between Hilbert spaces, our aim in this section is to provide sufficient conditions to ensure the prox-regularity of the set

$$
S=\left(F_{1}+F_{2}\right)^{-1}(0)=\left\{x \in \mathcal{H}: 0 \in F_{1}(x)+F_{2}(x)\right\} .
$$

Let us first observe that whenever one of the multimappings involved is single-valued, say $F_{1}:=f, S$ is the solution set of the generalized equation

$$
f(x)+F(x) \ni 0,
$$


where $F:=F_{2}$. The particular case when one of the multimappings involved is constant is also of interest. Indeed, if $F_{1} \equiv B$ for $B \subset \mathcal{H}^{\prime}$ and $F:=-F_{2}$, it can be easily checked that

$$
S=F^{-1}(B):=\{x \in \mathcal{H}: F(x) \cap B \neq \emptyset\} .
$$

We start with the following example which shows that even for $F_{1} \equiv 0$ or $F_{1}$ the Clarke subdifferential of a smooth function, the set $S$ given by (6.3) may fail to be prox-regular.

Example 6.1. Let us define $F: \mathbb{R}^{2} \rightrightarrows \mathbb{R}^{2}, f: \mathbb{R}^{2} \rightarrow \mathbb{R}$ by

$$
F(x, y)=\{(0,0)\} \quad \text { and } \quad f(x, y)=x y \quad \text { for all }(x, y) \in \mathbb{R}^{2} .
$$

The set $S=\left\{(x, y) \in \mathbb{R}^{2}: 0 \in f(x, y)+F(x, y)\right\}=\mathbb{R} \times\{0\} \cup\{0\} \times \mathbb{R}$ is clearly not prox-regular at $(0,0)$.

Another bad situation is provided by the function $g: \mathbb{R} \rightarrow \mathbb{R}$ defined, as in [2], by

$$
g(x):=\left\{\begin{array}{lll}
x^{6}\left(1-\cos \left(\frac{1}{x}\right)\right) & \text { if } & x \neq 0 \\
0 & \text { if } & x=0 .
\end{array}\right.
$$

It is straightforward to check that $g$ is $C^{2}$ on $\mathbb{R}$ with $g^{\prime}(0)=0$. For each $x \in \mathbb{R}$, set

$$
h(x):=\int_{0}^{x} g(t) \mathrm{d} t
$$

and observe that

$$
\left\{x \in \mathbb{R}: 0 \in \partial_{C} h(x)\right\}=\{x \in \mathbb{R}: g(x)=0\}=\{0\} \cup\left\{\frac{1}{2 k \pi}: k \in \mathbb{Z}\right\}
$$

is not prox-regular at 0 .

Given a Banach space $X$ and two functions $f, g: X \rightarrow \mathbb{R}$ finite at $x \in X$, it is readily seen that $\partial_{F} f(x) \subset$ $\partial_{F} g(x)$ whenever $f(x)=g(x)$ and $f \leq g$ near $x$. This and the first equality of (3.1) justify the following result.

Proposition 6.2. Let $X, Y$ be Banach spaces, $F_{1}, F_{2}: X \rightrightarrows Y$ be two multimappings, $S=$ $\left\{x \in X: 0 \in F_{1}(x)+F_{2}(x)\right\},(\bar{x}, \bar{y}) \in \operatorname{gph} F_{1} \cap \operatorname{gph}\left(-F_{2}\right)$. Assume that there exist a real $\gamma \geq 0$, a real $\delta>0$ such that for all $(x, y) \in B(\bar{x}, \delta) \times B(\bar{y}, \delta)$,

$$
d(x, S) \leq \gamma\left(d\left((x, y), \operatorname{gph} F_{1}\right)+d\left((x, y), \operatorname{gph}\left(-F_{2}\right)\right) .\right.
$$

Then, one has

$$
\partial_{F} d_{S}(\bar{x}) \times\{0\} \subset \gamma\left(\partial_{C} d_{\mathrm{gph} F_{1}}(\bar{x}, \bar{y})+\partial_{C} d_{\mathrm{gph}\left(-F_{2}\right)}(\bar{x}, \bar{y})\right)
$$

in particular

$$
N^{F}(S ; \bar{x}) \cap \mathbb{B}_{X^{\star}} \times\{0\} \subset N^{C}\left(\operatorname{gph} F_{1} ;(\bar{x}, \bar{y})\right) \cap \gamma \mathbb{B}_{(X \times Y)^{\star}}+N^{C}\left(\operatorname{gph}\left(-F_{2}\right) ;(\bar{x}, \bar{y})\right) \cap \gamma \mathbb{B}_{(X \times Y)^{\star}} .
$$

In order to provide an openness sufficient condition ensuring (6.4), the following lemma is needed.

Lemma 6.3. Let $X, Y$ be Banach spaces, $F_{1}, F_{2}: X \rightrightarrows Y$ be multimappings. Let $M_{F_{1}, F_{2}}$ be the multimapping defined by

$$
\begin{aligned}
M_{F_{1}, F_{2}}: X \times Y \rightrightarrows(X \times Y)^{2} & \rightrightarrows(x, y) \longmapsto-((x, y),(x, y))+\operatorname{gph} F_{1} \times \operatorname{gph} F_{2}
\end{aligned}
$$

The following hold:

(a) If $\operatorname{gph} M_{1}$, gph $M_{2}$ are normally $\omega(\cdot)$-regular for some nondecreasing function $\omega(\cdot): \mathbb{R}_{+} \rightarrow \mathbb{R}_{+}$with $\omega(0)=0$, then the set $\operatorname{gph} M_{F_{1}, F_{2}}$ is $2 \omega((1+\sqrt{2}) \cdot)$-normally-regular. 
If in addition,

$$
\omega(r)^{2}+\omega(s)^{2} \leq \omega\left(\sqrt{r^{2}+s^{2}}\right)^{2} \quad \text { for all } r, s \in \mathbb{R}_{+},
$$

then the set gph $M_{F_{1}, F_{2}}$ is in fact $\omega((1+\sqrt{2}) \cdot)$-normally-regular.

(b) If $X$ and $Y$ are Hilbert spaces and gph $F_{1}$, gph $F_{2}$ are $r$-prox-regular for some $\left.r \in\right] 0,+\infty[$, then the set gph $M_{F_{1}, F_{2}}$ is $\frac{r}{(1+\sqrt{2})^{2}}$-prox-regular.

Proof.

(a) Assume that gph $M_{1}$, gph $M_{2}$ are normally $\omega(\cdot)$-regular for some nondecreasing function $\omega(\cdot): \mathbb{R}_{+} \rightarrow \mathbb{R}_{+}$. Set $Z:=X \times Y$. Consider the continuous linear mapping $A: Z \rightarrow Z^{2}$ defined by

$$
A(x, y)=-((x, y),(x, y)) \text { for all }(x, y) \in Z \text {. }
$$

It is not difficult to check that $\|A\| \leq \sqrt{2}$. Let us define $\varphi: Z \times Z^{2} \rightarrow Z^{2}$ by

$$
\varphi((u, v), w)=w-A(u, v) \quad \text { for all }(u, v, w) \in Z^{3} .
$$

One observes that $\varphi$ is a continuous linear mapping with

$$
\|\varphi\| \leq 1+\|A\| \leq 1+\sqrt{2} .
$$

On the other hand, it is not difficult to check that gph $F_{1} \times \operatorname{gph} F_{2}$ is a normally $2 \omega(\cdot)$-regular subset of $Z^{2}$ (resp., normally $\omega(\cdot)$-regular of $Z^{2}$ if (6.5) holds true). According to Theorem 4.4, gph $M_{F_{1}, F_{2}}$ is a normally $2 \omega((1+\sqrt{2}) \cdot)$-regular subset of $Z^{2}$ (resp., normally $\omega((1+\sqrt{2}) \cdot)$-regular of $Z^{2}$ if (6.5) holds true).

(b) It is a direct consequence of $(a)$ with the function $\omega: \mathbb{R}_{+} \rightarrow \mathbb{R}_{+}$defined by

$$
\omega(t):=\frac{t^{2}}{2 r} \quad \text { for all } \quad t \in \mathbb{R}_{+},
$$

which obviously satisfies (6.5).

Thanks to the above result, we can apply Theorem 5.4 to the multimapping $M_{F_{1}, F_{2}}$.

Proposition 6.4. Let $X, Y$ be Banach spaces, $F_{1}, F_{2}: X \quad X \quad Y$ be multimappings, $S=$ $\left\{x \in X: 0 \in F_{1}(x)+F_{2}(x)\right\}$. Consider the following assumptions (a) and (b).

(a) There exists an upper semicontinuous nondecreasing function $\omega: \mathbb{R}_{+} \rightarrow \mathbb{R}_{+}$with

$$
\left.\omega(0)=0 \quad \text { and } \quad \omega(r)^{2}+\omega(s)^{2} \leq \omega\left(\sqrt{r^{2}+s^{2}}\right)^{2} \quad \text { for all } r, s \in\right] 0,+\infty[,
$$

and such that:

(i) gph $F_{1}$ and gph $F_{2}$ are normally $\omega(\cdot)$-regular subsets;

(ii) for $\rho(\cdot):=\omega((1+\sqrt{2}) \cdot)$, there exist $\alpha, \beta, s \in] 0,+\infty[$ with

$$
\beta>\frac{3 \alpha}{s}+\left(1+\frac{1}{s}\right) \rho\left(\sqrt{4 \alpha^{2}+\left(\beta-\frac{\alpha}{s}\right)^{2}}\right),
$$

and satisfying that for all $(\bar{x}, \bar{y}) \in \operatorname{gph} F_{1} \cap \operatorname{gph}\left(-F_{2}\right)$,

$$
\beta \mathbb{U}_{(X \times Y)^{2}} \subset-\left\{((x, y),(x, y)):(x, y) \in(\bar{x}, \bar{y})+\alpha \mathbb{B}_{X \times Y}\right\}+\operatorname{gph} F_{1} \times \operatorname{gph}\left(-F_{2}\right) .
$$


(b) The spaces $X$ and $Y$ are Hilbert and there exists a real $r \in] 0,+\infty]$ such that:

(i) gph $F_{1}$ and gph $F_{2}$ are $r$-prox-regular subsets of $X \times Y$;

(ii) there exist $\alpha, \beta, s \in] 0,+\infty[$ with

$$
\beta>\frac{3 \alpha}{s}+\frac{(1+\sqrt{2})^{2}}{2 r}\left(1+\frac{1}{s}\right)\left[4 \alpha^{2}+\left(\beta-\frac{\alpha}{s}\right)^{2}\right]
$$

and satisfying (6.6).

Under either $(a)$ or $(b)$, for each $(\bar{x}, \bar{y}) \in \operatorname{gph} F_{1} \cap \operatorname{gph}\left(-F_{2}\right)$, there exists a real $\delta>0$ such that for all $x \in B(\bar{x}, \delta)$ and for all $y \in B(\bar{y}, \delta)$,

$$
d(x, S) \leq 2 s\left[d\left((x, y), \operatorname{gph} F_{1}\right)+d\left((x, y), \operatorname{gph}\left(-F_{2}\right)\right)\right] .
$$

Proof. It suffices to show the result under $(a)$. So, assume that $(a)$ holds true. Endow $Z:=X \times Y$ with the 2-norm. For any subset $A \subset Z^{2}$, let us denote $d_{1}(\cdot, A)$ the distance function from $A$ associated to the 1-norm on $Z^{2}$. Put $M=M_{F_{1}, F_{2}}$, where

$$
\begin{aligned}
M_{F_{1}, F_{2}}: Z & \rightrightarrows Z^{2} \\
(x, y) & \longmapsto-((x, y),(x, y))+\operatorname{gph} F_{1} \times \operatorname{gph}\left(-F_{2}\right) .
\end{aligned}
$$

Thanks to $(i)$ of $(a)$ and Lemma 6.3 , we know that $\operatorname{gph} M$ is $\rho(\cdot)$-normally regular. Set

$$
Q=\left\{(x, y, 0, \ldots, 0):(x, y) \in \operatorname{gph} F_{1} \cap \operatorname{gph}\left(-F_{2}\right)\right\} \subset \operatorname{gph} M .
$$

Fix any $(\bar{x}, \bar{y}) \in\left(\operatorname{gph} F_{1}\right) \cap \operatorname{gph}\left(-F_{2}\right)$. Combining $(i i)$ of $(a)$ and Theorem 5.4, we know that there is a real $\delta_{0}>0$ such that for all $(x, y) \in B\left((\bar{x}, \bar{y}), \delta_{0}\right)$,

$$
d\left((x, y), M^{-1}(0, \ldots, 0)\right) \leq s d((0, \ldots 0), M(x, y)) .
$$

Note that

$$
M^{-1}(0, \ldots, 0)=\operatorname{gph} F_{1} \cap \operatorname{gph}\left(-F_{2}\right) \subset S \times Y .
$$

It is not difficult to check that for all $(x, y) \in Z$,

$$
\begin{aligned}
d((0, \ldots, 0), M(x, y)) & =d\left((x, y, x, y), \operatorname{gph} F_{1} \times \operatorname{gph}\left(-F_{2}\right)\right) \\
& \leq \sqrt{2} d_{1}\left((x, y, x, y), \operatorname{gph} F_{1} \times \operatorname{gph}\left(-F_{2}\right)\right) \\
& =\sqrt{2}\left[d\left((x, y), \operatorname{gph} F_{1}\right)+d\left((x, y), \operatorname{gph}\left(-F_{2}\right)\right)\right]
\end{aligned}
$$

In a similar way, we have for all $(x, y) \in Z$,

$$
\begin{aligned}
d\left((x, y), M^{-1}(0, \ldots, 0)\right) & =d\left((x, y), \operatorname{gph} F_{1} \cap \operatorname{gph}\left(-F_{2}\right)\right) \\
& \geq \frac{1}{\sqrt{2}} \inf \left\{\left\|x-x^{\prime}\right\|+\left\|y-y^{\prime}\right\|:\left(x^{\prime}, y^{\prime}\right) \in \operatorname{gph} F_{1} \cap \operatorname{gph}\left(-F_{2}\right)\right\} \\
& \geq \frac{1}{\sqrt{2}} \inf \left\{\left\|x-x^{\prime}\right\|+\left\|y-y^{\prime}\right\|:\left(x^{\prime}, y^{\prime}\right) \in S \times Y\right\} \\
& =\frac{1}{\sqrt{2}}\left[\inf \left\{\left\|x-x^{\prime}\right\|: x^{\prime} \in S\right\}+\inf \left\{\left\|y-y^{\prime}\right\|: y^{\prime} \in Y\right\}\right] \\
& =\frac{1}{\sqrt{2}} d(x, S),
\end{aligned}
$$

where the second inequality is due to the inclusion (6.8). As a consequence, we obtain for all $x \in B(\bar{x}, \delta)$, for all $y \in B(\bar{y}, \delta)$,

$$
d(x, S) \leq 2 s\left[d\left((x, y), \operatorname{gph} F_{1}\right)+d\left((x, y), \operatorname{gph}\left(-F_{2}\right)\right)\right] .
$$


With the above results at hand, we can prove the main theorem of this section:

Theorem 6.5. Let $X, Y$ be Banach spaces, $F_{1}, F_{2}: X \rightrightarrows Y$ be multimappings such that $S=$ $\left\{x \in \mathcal{H}: 0 \in F_{1}(x)+F_{2}(x)\right\}$ is nonempty and closed.

(A) Assume that (a) in Proposition 6.4 is satisfied and that there exist a selection $\sigma: S \rightarrow \mathcal{H}^{\prime}$ of $\left(-F_{1}\right) \cap F_{2}$ and a real $L \geq 0$ satisfying for all $x, x^{\prime} \in S$,

$$
\left\|\sigma(x)-\sigma\left(x^{\prime}\right)\right\| \leq L\left\|x-x^{\prime}\right\| .
$$

Then, the set $S$ is $4 s \omega\left(\left(1+L^{2}\right)^{\frac{1}{2}}\right.$.)-normally regular.

(B) Assume that (b) in Proposition 6.4 is satisfied and that there exists a selection $\sigma: S \rightarrow \mathcal{H}^{\prime}$ of $\left(-F_{1}\right) \cap F_{2}$ such that there exist $\rho \in] 0,+\infty]$ and a real $L \geq 0$ satisfying for all $x, x^{\prime} \in S$ with $\left\|x-x^{\prime}\right\|<2 \rho$,

$$
\left\|\sigma(x)-\sigma\left(x^{\prime}\right)\right\| \leq L\left\|x-x^{\prime}\right\| .
$$

Then, the set $S$ is $\min \left\{\rho, \frac{r}{4 s\left(1+L^{2}\right)}\right\}$-prox-regular.

Proof. Let us show $(A)$ (resp., $(B)$ ). Fix any $x, x^{\prime} \in S$ (resp., $x, x^{\prime} \in S$ with $\left.\left\|x-x^{\prime}\right\|<2 \rho\right)$ and $x^{\star} \in N^{F}(S ; x) \cap$ $\mathbb{B}_{X^{\star}}$. We have $\sigma(x) \in F_{2}(x) \cap\left(-F_{1}(x)\right)$ and $\sigma\left(x^{\prime}\right) \in F_{2}\left(x^{\prime}\right) \cap\left(-F_{1}\left(x^{\prime}\right)\right)$, in particular

$$
(x,-\sigma(x)),\left(x^{\prime},-\sigma\left(x^{\prime}\right)\right) \in \operatorname{gph} F_{1} \cap \operatorname{gph}\left(-F_{2}\right) .
$$

Combining Proposition 6.4 and Proposition 6.2, we get

$$
\left(N^{F}(S ; x) \cap \mathbb{B}_{X^{\star}}\right) \times\{0\} \subset N\left(\operatorname{gph} F_{1} ;(x,-\sigma(x))\right) \cap 2 s \mathbb{B}_{(X \times Y)^{\star}}+N\left(\operatorname{gph}\left(-F_{2}\right) ;(x,-\sigma(x))\right) \cap 2 s \mathbb{B}_{(X \times Y)^{\star} .}
$$

From the latter inclusion, there are $\left(u_{1}^{\star}, v_{1}^{\star}\right) \in N\left(\operatorname{gph} F_{1} ;(x,-\sigma(x))\right) \cap 2 s \mathbb{B}_{(X \times Y)^{\star}}$ and $\left(u_{2}^{\star}, v_{2}^{\star}\right) \quad \in$ $N\left(\operatorname{gph}\left(-F_{2}\right) ;(x,-\sigma(x))\right) \cap 2 s \mathbb{B}_{(X \times Y)^{\star}}$ such that

$$
\left(x^{\star}, 0\right)=\left(u_{1}^{\star}, v_{1}^{\star}\right)+\left(u_{2}^{\star}, v_{2}^{\star}\right) .
$$

Then, we can write

$$
\begin{aligned}
\left\langle x^{\star}, x^{\prime}-x\right\rangle & =\left\langle\left(x^{\star}, 0\right),\left(x^{\prime},-\sigma\left(x^{\prime}\right)\right)-(x,-\sigma(x))\right\rangle \\
& =\left\langle\left(u_{1}^{\star}, v_{1}^{\star}\right),\left(x^{\prime},-\sigma\left(x^{\prime}\right)\right)-(x,-\sigma(x))\right\rangle+\left\langle\left(u_{2}^{\star}, v_{2}^{\star}\right),\left(x^{\prime},-\sigma\left(x^{\prime}\right)\right)-(x,-\sigma(x))\right\rangle .
\end{aligned}
$$

Thanks to $(A)$ (resp., $(B))$, the latter equality entails

$$
\begin{aligned}
\left\langle x^{\star}, x^{\prime}-x\right\rangle & \leq 4 s \omega\left(\left\|\left(x^{\prime},-\sigma\left(x^{\prime}\right)\right)-(x,-\sigma(x))\right\|\right) \\
& \leq 4 s \omega\left(\left(1+L^{2}\right)^{\frac{1}{2}}\left\|x^{\prime}-x\right\|\right)
\end{aligned}
$$

(resp.,

$$
\begin{aligned}
\left\langle x^{\star}, x^{\prime}-x\right\rangle & \leq \frac{2 s}{r}\left(\left\|x^{\prime}-x\right\|^{2}+L^{2}\left\|x^{\prime}-x\right\|^{2}\right) \\
& =\frac{2 s\left(1+L^{2}\right)}{r}\left\|x^{\prime}-x\right\|^{2} .
\end{aligned}
$$

As a consequence, the set $S$ is $4 s \omega\left(\left(1+L^{2}\right)^{\frac{1}{2}} \cdot\right)$-normally regular (resp., $\min \left\{\rho, \frac{r}{4 s\left(1+L^{2}\right)}\right\}$-prox-regular).

Remark 6.6. Let $M(x):=\left(-F_{1}(x)\right) \cap F_{2}(x)$ and $S=\left\{x \in X: 0 \in F_{1}(x)+F_{2}(x)\right\}$. 
- One of the most important (and simple) case where $M(\cdot)$ admits a Lipschitz selection over $S$ is furnished with $F_{1}$ as a single-valued Lipschitz mapping.

- If $\operatorname{Dim} Y<+\infty$, according to ([4], Thm. 9.4.3), we know that $\left(-F_{1}\right) \cap F_{2}$ has a Lipschitz continuous selection whenever the two following conditions hold:

(a) $F_{1}, F_{2}$ are nonempty closed convex valued;

(b) $M:=\left(-F_{1}\right) \cap F_{2}$ is Lipschitz.

A particular case where $M(\cdot)$ is Lipschitz is obtained (see, [20], Prop. 1.22) with a Lipschitz multimapping $F_{2}$ over $S$ and with $F_{1}(x):=B[z(x), \rho(x)]$ provided that the mappings $z(\cdot), \rho(\cdot)$ are Lipschitz over $S$ and there is a real $0 \leq \delta<1$ satisfying

$$
B[z(x), \delta \rho(x)] \cap\left(-F_{2}(x)\right) \neq \emptyset \quad \text { for all } x \in S .
$$

- Let us mention that the concept of strong metric regularity (see, e.g., [13] and the references therein) which is a stronger version of metric regularity in the sense of the definition in (3.2) is linked with Lipschitz selections. Indeed, it is known (see [13]) that, for a multimapping $M: X \rightrightarrows Y$ between Banach spaces $X$ and $Y$ which is strongly metrically regular at $\bar{x}$ for $\bar{y}$ where $(\bar{x}, \bar{y}) \in \operatorname{gph} M$, one can find a Lipschitz selection of $M^{-1}(\cdot)$ defined near $\bar{y}$.

The case where one of the two multimappings involved in the latter theorem is single-valued leads to the following result.

Corollary 6.7. Let $\mathcal{H}, \mathcal{H}^{\prime}$ be Hilbert spaces, $f: \mathcal{H} \rightarrow \mathcal{H}^{\prime}$ be a mapping and $F: \mathcal{H} \rightrightarrows \mathcal{H}^{\prime}$ be a multimapping such that the set $S=\{x \in \mathcal{H}: 0 \in f(x)+F(x)\}$ is nonempty. Assume that:

(i) gph $F$ is $r$-prox-regular for some extended real $r \in] 0,+\infty]$;

(ii) the mapping $f$ is differentiable on $\mathcal{H}$ with $D f: \mathcal{H} \rightarrow \mathcal{H}^{\prime} \gamma$-Lipschitz on $\mathcal{H}$ for some real $\gamma \geq 0$ and there exist an extended real $\rho \in] 0,+\infty]$ and a real $L \geq 0$ such that for all $x, y \in S$, with $\|x-y\|<2 \rho$,

$$
\|f(x)-f(y)\| \leq L\|x-y\| ;
$$

(iii) there exist $\alpha, \beta, s \in] 0,+\infty[$ satisfying (6.7) and such that for all $\bar{x} \in S$,

$$
\beta \mathbb{U}_{\left(\mathcal{H} \times \mathcal{H}^{\prime}\right)^{2}} \subset-\left\{((x, y),(x, y)):(x, y) \in(\bar{x},-f(\bar{x}))+\alpha \mathbb{B}_{\mathcal{H} \times \mathcal{H}^{\prime}}\right\}+\operatorname{gph} F \times \operatorname{gph}(-f) .
$$

Then, the set $S$ is $r^{\prime}$-prox-regular with $r^{\prime}=\min \left\{\rho, \frac{\min \left\{r, \frac{1}{\gamma}\right\}}{4 s\left(L^{2}+1\right)}\right\}$.

Proof. Thanks to the continuity of $f$ and the fact that gph $M$ is closed, it is clear that $S$ is closed. Let us define $F_{1}, F_{2}: \mathcal{H} \rightrightarrows \mathcal{H}^{\prime}$ by

$$
F_{1}(x)=F(x) \quad \text { and } \quad F_{2}(x)=\{f(x)\} \quad \text { for all } x \in \mathcal{H} .
$$

According to Theorem 4.4 or Remark 4.6, gph $F_{2}$ is $\frac{1}{\gamma}$-prox-regular. It remains to apply Theorem 6.5 with the selection $\sigma=f_{\mid S}: S \rightarrow \mathcal{H}^{\prime}$ of $-F_{1} \cap F_{2}$.

\section{Preservation of the PROX-REgularity UNDER VARIOUS SET OPERATIONS}

This section is devoted to provide new results for the stability of prox-regularity under set operations, namely, inverse image, direct image and intersection. We also give sufficient conditions ensuring the prox-regularity of set constraints. For another previous developments on that topic, we refer the reader to $[1,2,12,42,43]$.

Let $F: \mathcal{H} \rightrightarrows \mathcal{H}^{\prime}$ be a multimapping between Hilbert spaces and let $B$ be a uniform prox-regular subset of $\mathcal{H}^{\prime}$. Keeping in mind Example 5.3, we know that

$$
F^{-1}(B):=\{x \in \mathcal{H}: F(x) \cap B \neq \emptyset\}
$$

fails in general to be prox-regular even supposing that $F$ has its graph uniformly prox-regular. Nevertheless, we have the following result as a consequence of Theorem 6.5. 
Proposition 7.1. Let $\mathcal{H}, \mathcal{H}^{\prime}$ be Hilbert spaces, $F: \mathcal{H} \rightrightarrows \mathcal{H}^{\prime}$ be a multimapping, and $B$ be a subset of $\mathcal{H}^{\prime}$ such that the set

$$
F^{-1}(B)=\{x \in \mathcal{H}: F(x) \cap B \neq \emptyset\}
$$

is nonempty and closed. Assume that:

(i) the sets gph $F$ and $B$ are $r$-prox-regular for some extended real $r \in] 0,+\infty]$;

(ii) there exists $\sigma: F^{-1}(B) \rightarrow B$ a selection of $F$ such that there exist $\left.\left.\rho \in\right] 0,+\infty\right]$ and a real $L \geq 0$ such that for all $x, x^{\prime} \in F^{-1}(B)$ with $\left\|x-x^{\prime}\right\|<2 \rho$,

$$
\left\|\sigma(x)-\sigma\left(x^{\prime}\right)\right\| \leq L\left\|x-x^{\prime}\right\| ;
$$

(iii) there exist $\alpha, \beta, s \in] 0,+\infty[$ satisfying (6.7) and such that for all $(\bar{x}, \bar{y}) \in \operatorname{gph} F \cap(\mathcal{H} \times B)$,

$$
\beta \mathbb{U}_{\left(\mathcal{H} \times \mathcal{H}^{\prime}\right)^{2}} \subset-\left\{((x, y),(x, y)):(x, y) \in(\bar{x}, \bar{y})+\alpha \mathbb{B}_{\mathcal{H} \times \mathcal{H}^{\prime}}\right\}+\operatorname{gph} F \times(\mathcal{H} \times B) .
$$

Then, the set $F^{-1}(B)$ is $r^{\prime}$-prox-regular with $r^{\prime}=\min \left\{\rho, \frac{r}{4 s\left(1+L^{2}\right)}\right\}$.

Proof. Let us define $F_{1}, F_{2}: \mathcal{H} \rightrightarrows \mathcal{H}^{\prime}$ by

$$
F_{1}(x)=B \quad \text { and } \quad F_{2}(x)=-F(x) \quad \text { for all } x \in \mathcal{H} .
$$

It can be checked in a straightforward way that

$$
F^{-1}(B)=\left\{x \in \mathcal{H}: 0 \in F_{1}(x)+F_{2}(x)\right\}
$$

and this allows us to apply Theorem 6.5.

Remark 7.2. Unfortunately, in general, gph $F$ with $F(\cdot):=\partial_{C} f$ is not prox-regular. However, from Theorem 4.4 (or Rem. 4.6), it is clear that a function $f: \mathcal{H} \rightarrow \mathbb{R}$ twice differentiable with Lipschitz continuous second derivative has the graph of its Clarke subdifferential (or equivalently, the graph of its first derivative) uniformly prox-regular.

Coming back to the preservation of prox-regularity, we have the two following results which complement ([2], Thm. 3.5).

Corollary 7.3. Let $\mathcal{H}$ be a Hilbert space, $m, n \in \mathbb{N}, f_{1}, \ldots, f_{m+n}: \mathcal{H} \rightarrow \mathbb{R}$ be functions such that the set

$$
S=\left\{x \in \mathcal{H}: f_{1}(x) \leq 0, \ldots, f_{m}(x) \leq 0, f_{m+1}(x)=0, \ldots, f_{m+n}(x)=0\right\}
$$

is nonempty. Assume that:

(a) there exist $\rho \in] 0,+\infty], L \in[0,+\infty[$ such that for all $i \in\{1, \ldots, m+n\}$, for all $x, y \in S$ with $\|x-y\|<2 \rho$,

$$
\left|f_{i}(x)-f_{i}(y)\right| \leq L\|x-y\| ;
$$

(b) $f_{1}, \ldots, f_{m+n}$ are differentiable on $\mathcal{H}$ and there exists a real $\gamma \geq 0$ such that for all $i \in\{1, \ldots, m+n\}, \nabla f_{i}$ is $\gamma$-Lipschitz;

(c) there exist $\alpha, \beta, s \in] 0,+\infty[$ satisfying (6.7) and such that for all $\bar{x} \in S$,

$$
\begin{aligned}
\beta \mathbb{U}_{\left(\mathcal{H} \times \mathbb{R}^{m+n}\right)^{2}} \subset & -\left\{((x, y),(x, y)):(x, y) \in\left(\bar{x}, f_{1}(\bar{x}), \ldots, f_{m+n}(\bar{x})\right)+\alpha \mathbb{B}_{\mathcal{H} \times \mathbb{R}^{m+n}}\right\} \\
& +\mathcal{H} \times\left(\mathbb{R}_{-}\right)^{m} \times\left\{0_{\mathbb{R}^{n}}\right\} \times \operatorname{gph}\left(f_{1}, \ldots, f_{m+n}\right) .
\end{aligned}
$$

Then, the set $S$ is $r^{\prime}=\min \left\{\rho, \frac{1}{4 \sqrt{2} \gamma s\left(1+2 L^{2}\right)}\right\}$-prox-regular. 
Proof. Let us define $f: \mathcal{H} \rightarrow \mathbb{R}^{m+n}$ and $F: \mathcal{H} \rightrightarrows \mathbb{R}^{m+n}$ by

$$
f(x)=\left(-f_{1}(x), \ldots,-f_{m+n}(x)\right) \quad \text { and } \quad F(x)=\left(\mathbb{R}_{-}\right)^{m} \times\left\{0_{\mathbb{R}^{n}}\right\} \quad \text { for all } x \in \mathcal{H},
$$

where $\mathbb{R}_{-}:=-\mathbb{R}_{+}$. Denotes by $\|\cdot\|_{2}$ (resp., $\|\cdot\|_{\infty}$ ) the usual Euclidean (resp., supremum) norm on $\mathbb{R}^{m+n}$. Fix any $u, v \in S$ with $\|u-v\|<2 \rho$. Thanks to $(i)$, we observe that

$$
\begin{aligned}
\|f(u)-f(v)\|_{2} & \leq \sqrt{2}\|f(u)-f(v)\|_{\infty} \\
& =\sqrt{2}\left\|\left(f_{1}(u)-f_{1}(v), \ldots, f_{m+n}(u)-f_{m+n}(v)\right)\right\|_{\infty} \\
& \leq \sqrt{2} L\|u-v\| .
\end{aligned}
$$

In a similar way, we can show that $D f$ is $\sqrt{2} \gamma$-Lipschitz. Applying Corollary 6.7 , we know that the set

$$
\{x \in \mathcal{H}: 0 \in f(x)+F(x)\}=S
$$

is $\min \left\{\rho, \frac{1}{4 \sqrt{2} \gamma s\left(1+2 L^{2}\right)}\right\}$-prox-regular.

Unfortunately, as shown in the next example, a function with uniform prox-regular graph may fail to have its sublevels prox-regular. The following definition will be needed.

Definition 7.4. Let $\mathcal{H}$ be a Hilbert space, $C$ be a nonempty convex subset of $\mathcal{H}, f: C \rightarrow \mathbb{R} \cup\{+\infty\}$ be a function, $\sigma \geq 0$ be a real. One says that $f$ is $\sigma$-semiconvex whenever

$$
f(t x+(1-t) y) \leq \lambda f(x)+(1-\lambda) f(y)+\frac{1}{2} \sigma t(1-t)\|x-y\|^{2},
$$

for all $x, y \in C$, for all $t \in] 0,1\left[\right.$ or equivalently if $f+\frac{\sigma}{2}\|\cdot\|^{2}$ is convex on $C$.

It is known that a $\sigma$-semiconvex function on an open convex set $U$ has its Clarke subdifferential hypomonotone on $U$ (see, e.g., [2] and the references therein).

Example 7.5. Sublevel sets of funtions with prox-regular epigraphs may fail to be prox-regular.

Let us set $C:=\left[-\frac{1}{2}, \frac{1}{2}\right]$ and $\left.U:=\right]-1,1[$. Consider $g: \mathbb{R} \rightarrow \mathbb{R}$ defined by $g(0)=0$ and

$$
g(x)=x^{6}\left(1-\cos \left(\frac{1}{x}\right)\right) \quad \text { for all } \quad x \in \mathbb{R} \backslash\{0\},
$$

and $h: U \times \mathbb{R} \rightarrow \mathbb{R}$

$$
h(x, t):=g(x)-t \quad \text { for all }(x, t) \in U \times \mathbb{R} .
$$

Set $S:=\{(x, t) \in C \times \mathbb{R}: h(x, t) \leq 0\}$. One can see that $g$ is $C^{2}$ on $\mathbb{R}$ and $\gamma$-semiconvex on $U$ for $\gamma \geq 0$ large enough. From the definition of $h$, it is then clear that $h+\gamma^{\prime}\|(\cdot, \cdot)\|^{2}$ is convex on $U \times \mathbb{R}$ for $\gamma^{\prime} \geq \gamma$ large enough, in particular $h$ is semiconvex on $U \times \mathbb{R}$. On the other hand, we have

$$
\langle\nabla h(x, t),(0,1)\rangle=-1 \quad \text { for all }(x, t) \in U \times \mathbb{R} .
$$

Using the same arguments as in the proof of ([2], Thm. 4.1), we arrive to the fact that $S$ is uniformly proxregular. Hence, $S=$ epi $\hat{g}$ is uniformly prox-regular, where $\hat{g}: \mathbb{R} \rightarrow \mathbb{R} \cup\{+\infty\}$ is defined by $\hat{g}(x)=g(x)$ if $x \in C$ and $\hat{g}(x)=+\infty$ outside of $C$. On the other hand, we observe that the (zero) sublevel set of the function $\hat{g}$, say

$$
\{x \in \mathbb{R}: \hat{g}(x) \leq 0\}=\{0\} \cup\left\{\frac{1}{2 k \pi}: k \in \mathbb{Z}\right\},
$$

is not prox-regular while its epigraph is uniformly prox-regular. 
It is worth pointing out that one can even avoid the use of extended real-valued functions. Indeed, one can consider the real-valued function $g_{0}: \mathbb{R} \rightarrow \mathbb{R}$ defined by

$$
g_{0}(x):=\left\{\begin{array}{lll}
g(x) & \text { if } & x \in]-1,1[ \\
\varphi(x) & \text { if } & x>1 \\
\varphi(-x) & \text { if } & x<-1
\end{array}\right.
$$

where $\varphi(x):=a x^{2}+b x+c$ for all $x \in \mathbb{R}$ with $a:=(1 / 2) g^{\prime \prime}(1), b:=g^{\prime}(1)-2 a$ and $c:=g(1)-a-b$. It can be checked that $g_{0}$ has its second order derivative bounded over $\mathbb{R}$ and this allows us to apply the same arguments as above to get that epi $g_{0}$ is uniformly prox-regular and that its sublevel set $\left\{x \in \mathbb{R}: g_{0}(x) \leq 0\right\}$ fails to be prox-regular.

Despite the negative cases in the above example, the following corollary provides conditions for prox-regularity of sublevel sets of functions with prox-regular epigraphs.

Corollary 7.6. Let $\mathcal{H}$ be a Hilbert space, $C$ be a nonempty subset of $\mathcal{H}, f: C \rightarrow \mathbb{R} \cup\{+\infty\}$ be a function such that the set $\{f \leq 0\}$ is nonempty and epi $f$ is $r$-prox-regular for some extended real $r \in] 0,+\infty]$. Assume that there exist $\alpha, \beta, s \in] 0,+\infty[$ satisfying (6.7) and such that for all $\bar{x} \in\{f \leq 0\}$,

$$
\beta \mathbb{U}_{(\mathcal{H} \times \mathbb{R})^{2}} \subset-\left\{((x, t),(x, t)):(x, t) \in(\bar{x}, 0)+\alpha \mathbb{B}_{\mathcal{H} \times \mathbb{R}}\right\}+\text { epi } f \times(\mathcal{H} \times\{0\}) .
$$

Then, the set $\{f \leq 0\}$ is $\frac{r}{4 s}$-prox-regular.

Proof. Let us define $F: \mathcal{H} \rightrightarrows \mathbb{R}$ by

$$
F(x)= \begin{cases}\{r \in \mathbb{R}: f(x) \leq r\} & \text { if } \quad x \in C \\ \emptyset & \text { otherwise. }\end{cases}
$$

The multimapping $M$ obviously satisfies gph $F=$ epi $f$ and $M^{-1}(0)=\{f \leq 0\}$. It remains to apply Proposition 7.1 to complete the proof.

The two next results are concerned with the single-valued case. The first deals with the inverse image.

Corollary 7.7. Let $\mathcal{H}, \mathcal{H}^{\prime}$ be Hilbert spaces, $f: \mathcal{H} \rightarrow \mathcal{H}^{\prime}$ be a mapping, $B$ be a subset of $\mathcal{H}^{\prime}$ such that $f^{-1}(B)$ is nonempty and closed. Assume that:

(i) $\operatorname{gph} f$ and $B$ are $r$-prox-regular for some extended real $r \in] 0,+\infty]$;

(ii) there exists an extended real $\rho \in] 0,+\infty]$, a real $L \geq 0$ such that for all $x, x^{\prime} \in f^{-1}(B)$ with $\left\|x-x^{\prime}\right\|<2 \rho$,

$$
\left\|f(x)-f\left(x^{\prime}\right)\right\| \leq L\left\|x-x^{\prime}\right\| ;
$$

(iii) there exist $\alpha, \beta, s \in] 0,+\infty\left[\right.$ satisfying (6.7) and such that for all $\bar{x} \in f^{-1}(B)$,

$$
\beta \mathbb{U}_{\left(\mathcal{H} \times \mathcal{H}^{\prime}\right)^{2}} \subset-\left\{((x, y),(x, y)):(x, y) \in(\bar{x}, f(\bar{x}))+\alpha \mathbb{B}_{\mathcal{H} \times \mathcal{H}^{\prime}}\right\}+\operatorname{gph} f \times(\mathcal{H} \times B) .
$$

Then, the set $f^{-1}(B)$ is $\min \left\{\rho, \frac{r}{4 s\left(1+L^{2}\right)}\right\}$-prox-regular.

Remark 7.8. In ([2], Thm. 6.4), such a preservation of prox-regularity is established but under an openness condition (on the derivative of $f$ and the tangent cone of $B$ ) required on a truncated neighborhood of each point of bdry $B \times\{0\}$ (where bdry $B$ denotes the boundary of $B$ ). 
In [6], the authors introduced the set $Q$ in Example 5.3 and they observed that

$$
Q \cap(\mathbb{R} \times\{0\})
$$

is not prox-regular at $(0,0)$ (see Fig. 2). It is worth pointing out that Example 7.5 can also be used to illustrate this fact. Indeed, with notation of Example 7.5, we see that

$$
S \cap(\mathbb{R} \times\{0\})=\{(0,0)\} \cup\left\{\left(\frac{1}{2 k \pi}, 0\right): k \in \mathbb{N}\right\}
$$

is not prox-regular at $(0,0)$ while $S$ is uniformly prox-regular.

So, unlike the convexity, the property of prox-regularity is not preserved by intersection. However, under certain conditions on normal cones [12] or openness conditions on the tangent cones of the sets involved ([2]) such a preservation is guaranteed. Here, it is obtained as a consequence of the above result on the inverse image.

Proposition 7.9. Let $\mathcal{H}$ be a Hilbert space, $m \in \mathbb{N}, S_{1}, \ldots, S_{m}$ be r-prox-regular subsets of $\mathcal{H}$ for some extended real $r \in] 0,+\infty]$ such that $\bigcap_{i=1}^{m} S_{i} \neq \emptyset$. Assume that there exist $\left.\alpha, \beta, s \in\right] 0,+\infty[$ satisfying (6.7) and such that for all $\bar{x} \in \bigcap_{i=1}^{m} S_{i}$,

$$
\begin{aligned}
\beta \mathbb{U}_{\mathcal{H}^{2(m+1)}} \subset & -\left\{\left(\left(x_{1}, \ldots, x_{m+1}\right),\left(x_{1}, \ldots, x_{m+1}\right)\right):\left(x_{1}, \ldots, x_{m+1}\right) \in(\bar{x}, \ldots, \bar{x})+\alpha \mathbb{B}_{\mathcal{H}^{m+1}}\right\} \\
& +\Delta_{\mathcal{H}^{m+1}} \times \mathcal{H} \times \prod_{i=1}^{m} S_{i} .
\end{aligned}
$$

Then, the set $\bigcap_{i=1}^{m} S_{i}$ is $\frac{r}{4 s(1+m)}$-prox-regular.

Proof. Let us define the mapping $f: \mathcal{H} \rightarrow \mathcal{H}^{m}$ by

$$
f(x)=(x, \ldots, x) \text { for all } x \in \mathcal{H},
$$

which is obviously $\sqrt{m}$-Lipschitz continuous. Moreover, note that gph $f=\Delta_{\mathcal{H}^{m+1}}$ is $\infty$-prox-regular (i.e., nonempty closed and convex). Taking into account the fact that $\prod_{i=1}^{m} S_{i}$ is $r$-prox-regular, the following equality

$$
f^{-1}\left(\prod_{i=1}^{m} S_{i}\right)=\bigcap_{i=1}^{m} S_{i}
$$

allows us to apply Corollary 7.7. This completes the proof.

Given a mapping $f$ between Hilbert spaces and a prox-regular set $S$, our aim is now to develop sufficient conditions ensuring the prox-regularity of $f(S)$. The next example shows that even for $S$ convex and $f$ smooth, such a preservation may fail.

Example 7.10. Consider the mapping $f: \mathbb{R} \longrightarrow \mathbb{R}^{2}$ defined by

$$
f(t)=\left(\frac{\sin (t)}{1+\cos ^{2}(t)}, \frac{\sin (t) \cos (t)}{1+\cos ^{2}(t)}\right) \quad \text { for all } t \in \mathbb{R} .
$$

The range of $f$ is the Bernoulli's Lemniscate which is not prox-regular at $(0,0)$.

In the line of ([12], Prop. 37), we have the following result. 


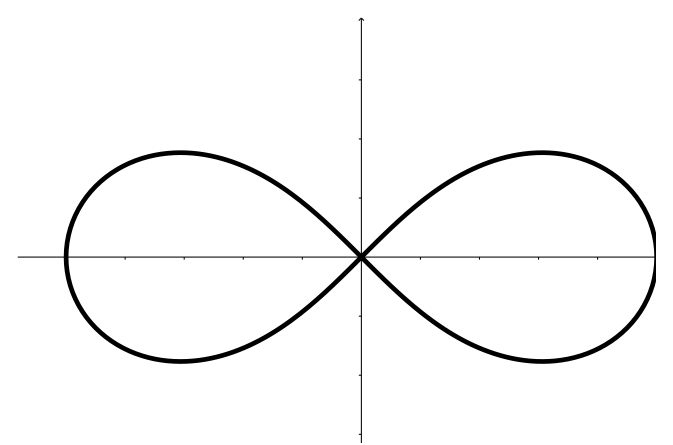

FiguRE 3. Bernoulli's Lemniscate.

Proposition 7.11. Let $\mathcal{H}, \mathcal{H}^{\prime}$ be Hilbert spaces, $f: \mathcal{H} \rightarrow \mathcal{H}^{\prime}$ be a mapping, $B$ be a subset of $\mathcal{H}$ such that $f(B)$ is closed. Assume that:

(a) gph $f$ and $B$ are $r$-prox-regular for some $r \in] 0,+\infty]$;

(b) there exists a mapping $\sigma: f(B) \rightarrow B$ satisfying $f \circ \sigma=\mathrm{Id}$ and for which there exist $\rho \in] 0,+\infty]$ and a real $L \geq 0$ such that for all $y, y^{\prime} \in f(B)$ with $\left\|y-y^{\prime}\right\|<2 \rho$,

$$
\left\|\sigma(y)-\sigma\left(y^{\prime}\right)\right\| \leq L\left\|y-y^{\prime}\right\| .
$$

(c) there exists $\alpha, \beta, s \in] 0,+\infty[$ satisfying (6.7) and such that for all $\bar{x} \in B$,

$$
\beta \mathbb{U}_{\left(\mathcal{H}^{\prime} \times \mathcal{H}\right)^{2}} \subset-\left\{((y, x),(y, x)):(y, x) \in(f(\bar{x}), \bar{x})+\alpha \mathbb{B}_{\mathcal{H}^{\prime} \times \mathcal{H}}\right\}+\{(f(x), x): x \in \mathcal{H}\} \times\left(\mathcal{H}^{\prime} \times B\right) .
$$

Then, the set $f(B)$ is $\min \left\{\rho, \frac{r}{4 s\left(1+L^{2}\right)}\right\}$-prox-regular.

Proof. Let us define the multimapping $F: \mathcal{H}^{\prime} \rightrightarrows \mathcal{H}$ by

$$
F(y)=\{x \in \mathcal{H}: y=f(x)\} \quad \text { for all } y \in \mathcal{H}^{\prime} .
$$

It is straightforward that

$$
F^{-1}(x):=\{f(x)\} \text { for all } x \in \mathcal{H},
$$

so in particular $F^{-1}(B)=f(B)$. Then, the prox-regularity claimed is a direct consequence of Proposition 7.1.

The assumption $(b)$ in the latter proposition leads for the preservation of prox-regularity under a direct image to develop a particular study depending on the given mapping $f$. We are going to illustrate this fact with the projection along a linear space. More precisely, given two Hilbert spaces $\mathcal{H}, \mathcal{H}^{\prime}$ and an $r$-prox-regular set $S$ in $\mathcal{H} \times \mathcal{H}^{\prime}$ for some $\left.\left.r \in\right] 0,+\infty\right]$, our aim is now to give sufficient condition to ensure the prox-regularity of $\Pi_{\mathcal{H}}(S)$, where $\Pi_{\mathcal{H}}: \mathcal{H} \times \mathcal{H}^{\prime} \rightarrow \mathcal{H}$ is the mapping defined by

$$
\Pi_{\mathcal{H}}(x, y)=x \quad \text { for all }(x, y) \in \mathcal{H} \times \mathcal{H}^{\prime} .
$$

Obviously, if $r=+\infty$, then $\Pi_{\mathcal{H}}(S)$ is convex (since $S$ itself is convex when $r=+\infty$ ). Let us first show that such a preservation fails for $r<+\infty$.

Example 7.12. We are going to construct a sequence $\left(\mathcal{C}_{n}\right)_{n \in \mathbb{N}}$ of closed balls of $\mathbb{R}^{2}$ such that the set $\bigcup_{n \in \mathbb{N}} \mathcal{C}_{n}$ is uniformly prox-regular and $\Pi\left(\bigcup_{n \in \mathbb{N}} \mathcal{C}_{n}\right)$ is closed but not prox-regular, where $\Pi: \mathbb{R}^{2} \rightarrow \mathbb{R}$ is the mapping defined by

$$
\Pi(x, y)=x \quad \text { for all }(x, y) \in \mathbb{R}^{2} .
$$


Fix any sequence $\left(\alpha_{n}\right)_{n \geq 1}$ of $] 0, \frac{\pi}{2}\left[\right.$ with $\alpha_{n} \rightarrow 0$ and $\sum_{n=1}^{+\infty} \alpha_{n}=+\infty$. Let $\left.\delta \in\right] 0,+\infty\left[\right.$ and $\left(x_{1}, y_{1}\right) \in \mathbb{R}^{2}$. Choose any real $r_{1}$ such that

$$
0<r_{1}<\frac{\delta \sin \left(\alpha_{1}\right)}{1-\sin \left(\alpha_{1}\right)}
$$

From the latter inequality, we get

$$
0<\left(r_{1}+\delta\right) \sin \left(\alpha_{1}\right)-r_{1}
$$

so we can choose a real $r_{2}$ such that

$$
0<r_{2}<\min \left\{\left(r_{1}+\delta\right) \sin \left(\alpha_{1}\right)-r_{1}, \frac{\delta \sin \left(\alpha_{2}\right)}{1-\sin \left(\alpha_{2}\right)}\right\}
$$

It is then straightforward that we can construct a sequence $\left(r_{n}\right)_{n \geq 1}$ of reals such that

$$
0<r_{n}<\min \left\{\left(r_{n-1}+\delta\right) \sin \left(\alpha_{n-1}\right)-r_{n-1}, \frac{\delta \sin \left(\alpha_{n}\right)}{1-\sin \left(\alpha_{n}\right)}\right\} .
$$

Let us define $\left(x_{n}, y_{n}\right)_{n \in \mathbb{N}}$ by setting for all integer $n \geq 2$,

$$
x_{n}=x_{n-1}+\left(r_{n-1}+\delta+r_{n}\right) \sin \left(\alpha_{n-1}\right)
$$

and

$$
y_{n}=y_{n-1}-\left(r_{n-1}+\delta+r_{n}\right) \cos \left(\alpha_{n-1}\right) .
$$

For each $n \in \mathbb{N}$, denote by $\mathcal{C}_{n}$ the closed ball of $\mathbb{R}^{2}$ centered at $\left(x_{n}, y_{n}\right)$ with radius $r_{n}$. Set $\mathcal{C}=\bigcup_{n \in \mathbb{N}} \mathcal{C}_{n}$. It is readily seen that

$$
\Pi\left(\mathcal{C}_{n}\right)=\left[x_{n}-r_{n}, x_{n}+r_{n}\right] \text { for all } n \in \mathbb{N},
$$

so in particular

$$
\Pi(\mathcal{C})=\bigcup_{n \in \mathbb{N}}\left[x_{n}-r_{n}, x_{n}+r_{n}\right]
$$

Moreover, we have for all integer $n \geq 2$,

$$
\begin{aligned}
\left(x_{n}-r_{n}\right)-\left(x_{n-1}+r_{n-1}\right) & =\left(r_{n-1}+\delta+r_{n}\right) \sin \left(\alpha_{n-1}\right)-\left(r_{n}+r_{n-1}\right) \\
& >\left(r_{n-1}+\delta\right) \sin \left(\alpha_{n-1}\right)-\left(r_{n}+r_{n-1}\right) \\
& >0
\end{aligned}
$$

where the last inequality is due to the definition of $r_{n}$. Further, we have $r_{n} \rightarrow 0$ (thanks to the fact that $\alpha_{n} \rightarrow 0$ ) and then

$$
\left(x_{n}-r_{n}\right)-\left(x_{n-1}+r_{n-1}\right) \rightarrow 0 .
$$

Obviously, this entails that $\Pi(\mathcal{C})$ is not prox-regular (see also Fig. 4). On the other hand, it can be checked in a straightforward way that (see again Fig. 4$), \mathcal{C}$ is $\frac{\delta}{2}$-prox-regular. It remains to show that $\Pi(\mathcal{C})$ is closed. Observe first that

$$
x_{n}-x_{n-1} \sim \delta \alpha_{n-1},
$$

so $\sum_{n=2}^{+\infty}\left(x_{n}-x_{n-1}\right)=+\infty$ (keeping in mind that $\left.\sum_{n=1}^{+\infty} \alpha_{n}=+\infty\right)$ and then it follows $x_{n} \rightarrow+\infty$. Fix any $\bar{x} \in \operatorname{cl} \Pi(\mathcal{C})$. There is a sequence $\left(u_{n}\right)_{n \in \mathbb{N}}$ of $\Pi(\mathcal{C})$ such that $u_{n} \in \Pi(\mathcal{C})$ for all $n \in \mathbb{N}$. By contradiction, assume that $\bar{x} \notin \Pi(\mathcal{C})$, so $\bar{x} \in] x_{1}+r_{1},+\infty\left[\right.$. Combining this inclusion with $x_{n}+r_{n} \rightarrow+\infty$, we know that the set

$$
A:=\left\{n \in \mathbb{N}: x_{n}+r_{n}<\bar{x}\right\}
$$




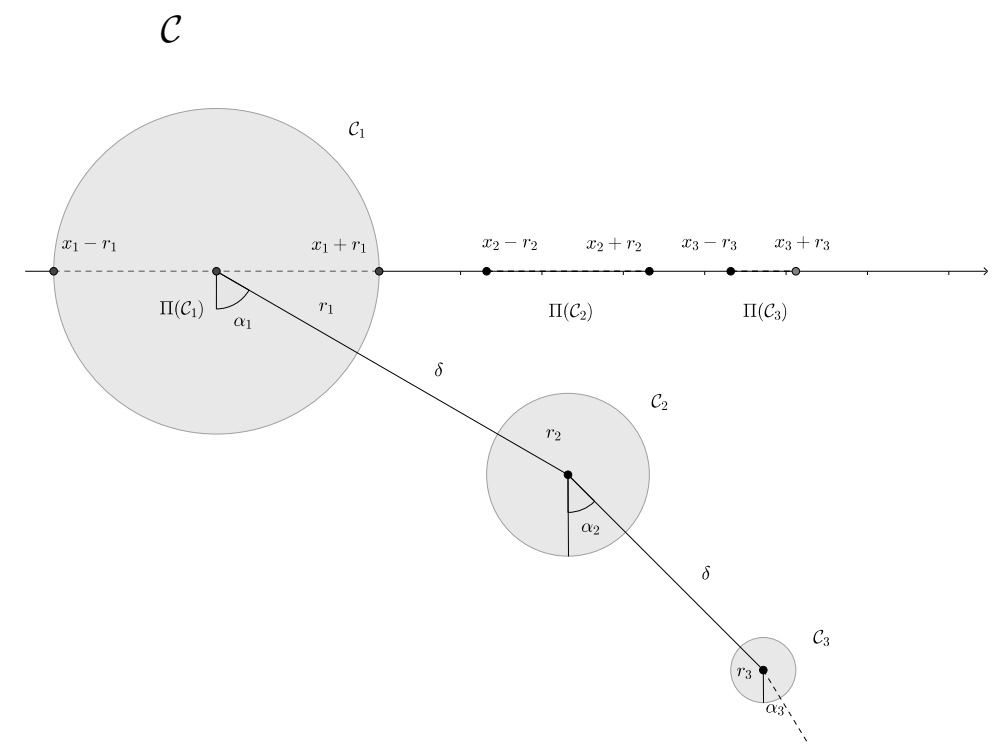

FiguRE 4. Projection of a prox-regular set which fails to be prox-regular.

is nonempty and finite. Let us set $N:=\max A$. Thanks to the definition of $N$, we have

$$
\bar{x} \in] x_{N}+r_{N}, x_{N+1}+r_{N+1}[.
$$

Keeping in mind that $\bar{x} \notin \Pi(\mathcal{C}) \supset\left[x_{N+1}-r_{N+1}, x_{N+1}+r_{N+1}\right]$, the inclusion above gives

$$
\bar{x} \in] x_{N}+r_{N}, x_{N+1}-r_{N+1}[
$$

and this cannot hold true since it entails that $u_{n} \notin \Pi(\mathcal{C})$ for $n$ large enough. As a consequence, the set $\Pi(\mathcal{C})$ is closed.

Before giving a necessary and sufficient condition ensuring the preservation of uniform prox-regularity under a projection along a vector space, let us come back to the problem of the prox-regularity of Dom $M$ for a multimapping $M$ with the prox-regular graph.

Example 7.13. With the notation of Example 7.12, let us consider the multimapping $M: \mathbb{R} \rightrightarrows \mathbb{R}$ defined by

$$
x \mapsto \begin{cases}\left\{y_{n}\right\} & \text { if } \quad x=x_{n} \quad \text { for some } \quad n \in \mathbb{N} \\ \emptyset & \text { otherwise. }\end{cases}
$$

It is clear that

$$
\operatorname{gph} M=\left\{\left(x_{n}, y_{n}\right): n \in \mathbb{N}\right\}
$$

is $\frac{\delta}{2}$-prox-regular. Since $x_{n}-x_{n-1} \rightarrow 0$, the set Dom $M=\left\{x_{n}: n \in \mathbb{N}\right\}$ is not prox-regular.

Before stating the last result of the paper, let us recall that a nonempty subset $S$ of a normed space $X$ is called proximinal whenever $\operatorname{Proj}_{S}(x) \neq \emptyset$ for all $x \in X$. If $X$ is reflexive, every nonempty weakly closed set in $X$ is known (and easily seen) to be proximinal. Recall also (see, e.g., [12]) that a nonempty weakly closed subset of $S$ of a Hilbert space is $r$-prox-regular for some extended real $r \in] 0,+\infty]$ if and only if $P_{S}$ is merely well defined in the open $r$-enlargement $U_{r}(S)$. 
Theorem 7.14. Let $\mathcal{H}, \mathcal{H}^{\prime}$ be Hilbert spaces, $S$ be an r-prox-regular set of $\mathcal{H} \times \mathcal{H}^{\prime}$ with $\left.\left.r \in\right] 0,+\infty\right]$, $\Pi_{\mathcal{H}}$ : $\mathcal{H} \times \mathcal{H}^{\prime} \rightarrow \mathcal{H}$ the mapping defined by

$$
\Pi_{\mathcal{H}}(x, y)=x \quad \text { for } \operatorname{all}(x, y) \in \mathcal{H} \times \mathcal{H}^{\prime} .
$$

If $\Pi_{\mathcal{H}}(S)$ is weakly closed, then $\Pi_{\mathcal{H}}(S)$ is r-prox-regular if and only if for each $x \in \mathcal{H}$ with $d_{\Pi_{\mathcal{H}}(S)}(x)<r$, there exists $w_{x} \in \mathcal{H}^{\prime}$ such that for all $v \in \operatorname{Proj}_{\Pi_{\mathcal{H}}(S)}(x),\left(v, w_{x}\right) \in S$.

Proof. Assume that $S^{\prime}:=\Pi_{\mathcal{H}}(S)$ is weakly closed.

$\Rightarrow$, Suppose that $S^{\prime}$ is $r$-prox-regular and fix any $x \in U_{r}\left(S^{\prime}\right)$. By the $r$-prox-regularity of $S^{\prime}$, we have

$$
\operatorname{Proj}_{S^{\prime}}(\bar{x})=\left\{\mathrm{P}_{S^{\prime}}(\bar{x})\right\} \text {. }
$$

Since $\mathrm{P}_{S^{\prime}}(\bar{x}) \in S^{\prime}$, there exist $(v, w) \in S$ such that $\mathrm{P}_{S^{\prime}}(\bar{x})=\Pi_{\mathcal{H}}(v, w)=v$. It follows that

$$
\left(\mathrm{P}_{S^{\prime}}(\bar{x}), w\right) \in S,
$$

which justifies the implication $\Rightarrow$. $\Leftarrow$, Let us prove the converse. Fix any $\bar{x} \in U_{r}\left(S^{\prime}\right)$ and fix also $v_{1}, v_{2} \in$ $\operatorname{Proj}_{S^{\prime}}(\bar{x}) \neq \emptyset$ (thanks to the fact that $S^{\prime}$ is proximinal). By assumption, there is $\bar{w}:=w_{\bar{x}} \in \mathcal{H}^{\prime}$ such that

$$
\left(v_{1}, \bar{w}\right) \in S \quad \text { and } \quad\left(v_{2}, \bar{w}\right) \in S .
$$

Fix any $i \in\{1,2\}$. We claim that $\left(v_{i}, \bar{w}\right) \in \operatorname{Proj}_{S}(\bar{x}, \bar{w})$. Observe first that

$$
d_{S^{\prime}}(\bar{x})=\left\|\bar{x}-v_{i}\right\|=\left\|(\bar{x}, \bar{w})-\left(v_{i}, \bar{w}\right)\right\| \geq d_{S}(\bar{x}, \bar{w}) .
$$

By contradiction, suppose that

$$
d_{S^{\prime}}(\bar{x})>d_{S}(\bar{x}, \bar{w})
$$

This inequality gives $(a, b) \in S$ such that

$$
\|(a, b)-(\bar{x}, \bar{w})\|<d_{S^{\prime}}(\bar{x})=\left\|(\bar{x}, \bar{w})-\left(v_{i}, \bar{w}\right)\right\|,
$$

and this entails

$$
d_{S^{\prime}}^{2}(\bar{x})>\|\bar{x}-a\|^{2}+\|\bar{w}-b\|^{2} \geq\|\bar{x}-a\|^{2} .
$$

On the other hand (thanks to the inclusion $a \in S^{\prime}$ ) we have

$$
\|\bar{x}-a\|^{2} \geq d_{S^{\prime}}^{2}(\bar{x}) .
$$

Combining (7.1) and (7.2), we get the desired contradiction and then we have

$$
d_{S}(\bar{x}, \bar{w})=d_{S^{\prime}}(\bar{x})=\left\|(\bar{x}, \bar{w})-\left(v_{i}, \bar{w}\right)\right\| .
$$

Since $\left(v_{i}, \bar{w}\right) \in S$, it results that

$$
\left(v_{i}, \bar{w}\right) \in \operatorname{Proj}_{S}(\bar{x}, \bar{w})
$$

Furthermore, note that

$$
d_{S}(\bar{x}, \bar{w})=d_{S^{\prime}}(\bar{x})<r,
$$

i.e., $(\bar{x}, \bar{w}) \in U_{r}(S)$. Since $S$ is $r$-prox-regular, the latter inclusion yields that $\operatorname{Proj}_{S}(\bar{x}, \bar{w})$ is a singleton. From (7.3), we have $v_{1}=v_{2}$ and then $P_{S}^{\prime}(\bar{x})$ is well defined. As a consequence, $S^{\prime}$ is $r$-prox-regular and this completes the proof. 


\section{CONCLUding REMARKS}

In this paper, the concept of a set normally $\omega(\cdot)$-regular is introduced. Such a class of sets encompasses prox-regular sets in Hilbert spaces and $(\sigma, \delta)$-subsmooth sets in general Banach spaces. Then, we provided a Robinson-Ursescu type theorem for multimappings with graph normally $\omega(\cdot)$-regular ensuring a kind of uniform metric regularity and we gave an estimation of the modulus. Finally, we established new results on the preservation of prox-regularity by set operations. The key assumption in Theorem 6.5 is the graph proxregularity of the multimappings involved in the problem. Many investigations could be achieved and particularly when one of the multimapping coincides with the subdifferential of a proper and lower-semicontinuous function. Due to their large number of applications, the prox-regularity of solution mappings to generalized equations involving the subdifferential of a nonconvex function is an important issue and will be the subject of a future research project.

\section{REFERENCES}

[1] S. Adly, F. Nacry and L. Thibault, Discontinuous sweeping process with prox-regular sets. ESAIM: COCV 23 (2017) 12931329.

[2] S. Adly, F. Nacry and L. Thibault, Preservation of prox-regularity of sets with applications to constrained optimization. SIAM J. Optim. 26 (2016) 448-473.

[3] J.-P. Aubin and A. Cellina, Differential inclusions. Set-valued maps and viability theory. Grundlehren der Mathematischen Wissenschaften. Springer Verlag, Berlin 264 (1984).

[4] J.-P. Aubin and H. Frankowska, Set-Valued Analysis, Birkhäuser Boston, Inc., Boston, MA (2009).

[5] D. Aussel, A. Daniilidis and L. Thibault, Subsmooth sets: functional characterizations and related concepts. Trans. Amer. Math. Soc. 357 (2005) 1275-1301.

[6] F. Bernard, L. Thibault and N. Zlateva, Prox-regular sets and epigraphs in uniformly convex Banach spaces: various regularities and other properties. Trans. Amer. Math. Soc. 363 (2011) 2211-2247.

[7] M. Bounkhel and D. Bounkhel, Inégalités variationnelles non convexes. ESAIM: COCV 11 (2005) 574-594.

[8] H. Brézis, Equations et inéquations non linéaires dans les espaces vectoriels en dualité. Ann. Inst. Fourier 18 (1968) $115-175$.

[9] P. Cannarsa and C. Sinestrari, Semiconcave functions, Hamilton-Jacobi equations, and optimal control, Birkhäuser (2004).

[10] F. Chazal, D. Cohen-Steiner and Q. Mérigot, Boundary measures for geometric inference. Found. Comput. Math. Vol. 10 (2010) 221-240.

[11] F.H. Clarke, Optimization and Nonsmooth Analysis, 2nd Edition. Classics in Applied Mathematics. Soc. Industrial Appl. Math. (SIAM), Philadelphia, PA 5 (1990).

[12] G. Colombo and L. Thibault, Prox-regular sets and applications, Handbook of nonconvex analysis and applications. Int. Press, Somerville, MA (2010) 99-182.

[13] A.L. Dontchev and R.T. Rockafellar, Implicit Functions and Solution Mappings. A view from variational analysis, 2nd edition. Springer Series in Operations Research and Financial Engineering. Springer, New York (2014).

[14] G. Duvaut and J.-L. Lions, Inequalities in mechanics and physics. Grundlehren der Mathematischen Wissenschaften. Springer Verlag, Berlin, New York 219 (1976).

[15] H. Federer, Curvature measures. Trans. Amer. Math. Soc. 93 (1959) 418-491.

[16] H. Federer, Geometric measure theory. Die Grundlehren der mathematischen Wissenschaften. Band 153 (1969) xiv+676.

[17] H. Huang and R.X. Li, Global error bounds for $\gamma$-paraconvex multifunctions. Set-Valued Var. Anal. 19 (2011) $487-504$.

[18] A. Jourani, Open mapping theorem and inversion theorem for $\gamma$-paraconvex multivalued mappings and applications. Studia Math. 117 (1996) 123-136.

[19] A. Jourani and E. Vilches, Positively $\alpha$-far sets and existence results for generalized perturbed sweeping processes. J. Convex Anal. 23 (2016) 775-821.

[20] J. Lindenstrauss and Y. Benyamini, Geometric nonlinear functional analysis, Vol. 1, American Mathematical Society Colloquium Publications. Amer. Math. Soc. Providence, RI 48 (2000).

[21] J.-L. Lions and G. Stampacchia, Variational inequalities. Commun. Pure Appl. Math. 20 (1967) $493-519$.

$[22]$ D.R. Luke, Finding best approximation pairs relative to a convex and prox-regular set in a Hilbert space. SIAM J. Optim. 19 (2008) 714-739.

[23] S. Marcellin and L. Thibault, Evolution problems associated with primal lower nice functions. J. Convex Anal. 13 (2006) 385-421.

[24] B. Maury and J. Venel, A discrete contact model for crowd motion. ESAIM: M2AN 45 (2011) 145-168.

[25] B. Maury, Nonsmooth evolution models in crowd dynamics: mathematical and numerical issues Collective dynamics from bacteria to crowds. CISM Courses Lect. 553 (2014) 47-73.

[26] B.S. Mordukhovich, Variational Analysis and Generalized Differentiation I. Grundlehren Series Vol. 330, Springer (2006).

[27] J.-J. Moreau, Rafle par un convexe variable I. Travaux Sém. Anal. Convexe Montpellier (1971), Exposé 15. 
[28] U. Mosco, Convergence of convex sets and of solutions of variational inequalities. Adv. Math. 3 (1969) $510-585$.

[29] J. Noel, Inclusions différentielles d'évolution associées à des ensembles sous-lisses. Thèse de doctorat (2013) Université Montpellier 2.

[30] R.A. Poliquin, Integration of subdifferentials of nonconvex functions. Nonl. Anal. 17 (1991) 385-398.

[31] R.A. Poliquin, R.T. Rockafellar and L. Thibault, Local differentiability of distance functions. Trans. Amer. Math. Soc. 352 (2000) 5231-5249.

[32] S.M. Robinson, Generalized equations and their solutions, Part I: Basic theory. Math. Programming Stud. (1979) 128-141.

[33] S.M. Robinson, Generalized equations and their solutions, Part II: Applications to nonlinear programming. Math. Programming Stud. (1982) 200-221.

[34] S.M. Robinson, Generalized equations. Mathematical programming: the state of the art. Bonn (1982) Springer, Berlin (1983) 346-367.

[35] S.M. Robinson, Regularity and stability for convex multivalued functions. Math. Oper. Res. 1 (1976) $130-143$.

[36] R.T. Rockafellar and R.J-B. Wets, Variational Analysis. Grundlehren der Mathematischen Wissenschaften. Springer, New York 317 (1998).

[37] S. Rolewicz, On paraconvex multifunctions. Oper. Res. Verfahren 31 (1979) 539-546.

[38] O.-S. Serea, On reflecting boundary problem for optimal control. SIAM J. Control Optim. 42 (2003) 559-575.

[39] G. Stampacchia, Formes bilinéaires coercitives sur les ensembles convexes. C. R. Acad. Sci. Paris 258 (1964) $4413-4416$.

[40] C. Thäle, 50 years sets with positive reach: a survey. Surv. Math. Appl. 3 (2008) 123-165.

[41] C. Ursescu, Multifunctions with closed convex graphs. Czechoslovak Math. J. 25 (1975) 438-411.

[42] J. Venel, A numerical scheme for a class of sweeping processes. Numer. Math. 118 (2011) 367-400.

[43] J.-P. Vial, Strong and weak convexity of sets and functions. Math. Oper. Res. 8 (1983) 231-259.

[44] X.Y. Zheng and Q.H. He, Characterization for metric regularity for $\sigma$-subsmooth multifunctions. Nonl. Anal. 100 (2014) $111-121$.

[45] X.Y. Zheng and K.F. Ng, Calmness for L-subsmooth multifunctions in Banach spaces. Siam J. Optim. 19 (2008) 1648-1673.

[46] X.Y. Zheng and K.F. Ng, Metric subregularity and calmness for nonconvex generalized equations in Banach spaces. Siam J. Optim. 20 (2010) 2119-2136.

[47] X.Y. Zheng and K.F. Ng, Metric Subregularity for proximal generalized equations in Hilbert spaces. Nonl. Anal. 75 (2012) $1686-1699$. 